IFT-UAM/CSIC-03-42

hep-th/yymmn

\title{
Flux-induced SUSY-breaking soft terms
}

\author{
P. G. Cámara*, L. E. Ibáñez*,** and A. M. Uranga* \\ * Departamento de Física Teórica C-XI and Instituto de Física Teórica C-XVI, \\ Universidad Autónoma de Madrid, Cantoblanco, 28049 Madrid, Spain. \\ ** Theory Division, CERN, 1211 Geneva 23, Switzerland.
}

\begin{abstract}
We describe the computation of SUSY-breaking terms on a D3-brane in a quite general type IIB supergravity background. We apply it to study the SUSY-breaking induced on the D3-brane worldvolume by the presence of NSNS and RR 3-form fluxes. We provide explicit general formulae for the SUSY-breaking soft terms valid for the different types of fluxes, leading to different patterns of soft terms. Imaginary anti self-dual fluxes with $G_{3}$ a pure $(3,0)$-form lead to soft terms corresponding to dilaton-dominated SUSY-breaking. More general SUSY-breaking patterns are discussed, arising from more general fluxes, or from distant anti-D3-branes. The known finiteness properties of dilaton-dominated soft terms are understood in terms of holography. The above results are interpreted in the context of the $4 \mathrm{~d}$ effective supergravity theory, where flux components correspond to auxiliary fields of e.g. the $4 \mathrm{~d}$ dilaton and overall volume modulus. We present semirealistic Type IIB orientifold examples with (meta)stable vacua leading to non-vanishing soft terms of the dilaton-domination type. Such models have many of the ingredients of the recent construction of de Sitter vacua in string theory. We finally explore possible phenomenological applications of this form of SUSY-breaking, where we show that soft terms are of order $M_{s}^{2} / M_{p}$. Thus a string scale of order $M_{s}=10^{10} \mathrm{GeV}$, and compactification scale three orders of magnitude smaller could explain the smallness of the weak scale versus the Planck mass.
\end{abstract}




\section{Introduction}

Low energy $\mathcal{N}=1$ supersymmetry is one of the most promising avenues to understand the stability of the electroweak scale against radiative corrections. Supersymmetry must however be broken, producing masses for the unobserved SUSY partners of quarks and leptons not much above the electroweak scale. One usually parametrizes supersymmetry breaking in terms of SUSY-breaking soft terms, i.e. $\operatorname{dim}<4$ operators which do not induce new quadratic divergences which would spoil the nice loop properties of $\mathcal{N}=1$ supersymmetry. These soft terms result after spontaneous supersymmetry breaking, irrespective of what the specific source of SUSY-breaking may be.

On the other hand, the structure of soft terms has a crucial impact on the pattern of masses and couplings for the SUSY partners of the usual Standard Model (SM) particles, and hence it would be very important to have a theory which makes predictions about these quantities. If low-energy SUSY is discovered at LHC such a theory would be amenable to direct experimental test.

If string theory is indeed the fundamental theory underlying both the gravitational as well as the SM interactions of elementary particles, it should be able to describe the structure of soft terms. Indeed, attempts to understand the structure of soft terms resulting from SUSY-breaking in perturbative heterotic compactifications were done since the early days of heterotic string phenomenology [1]. A general problem in addressing the computation of SUSY-breaking soft masses is the model-dependence of the results. In $[2,3,4]$ a slightly more model-independent approach was suggested 1. In most heterotic compactifications there are chiral fields corresponding to the complex dilaton $S$ and Kähler moduli $T^{i}$, whose (Planck mass suppressed) couplings to the charged fields are rather model-independent. The idea is to assume that the corresponding auxiliary fields $F^{S}, F^{i}$ get a vev, breaking SUSY. Even without knowing the microscopic source of this SUSY-breaking, the model-independence of the couplings of these fields allows to obtain specific predictions for soft terms, under the assumption of a vanishing cosmological constant. On the other hand a shortcoming of this approach is precisely that the specific mechanism of SUSY-breaking remains unexplained. In addition the prediction for the scalar soft masses does depend on the tuning of the cosmological constant.

In the last five years there have appeared several new ingredients in string theory

\footnotetext{
${ }^{1}$ An analogous analysis for compact Type IIB orientifolds was given in [5].
} 
which warrant a revision of these old problems. Specific Type II and Type I compactifications have been built with realistic particle spectrum. In these models the SM fields live in the worldvolume of D-branes embedded in a 10d closed string background $[6,7,8,9,10,11]$. Concerning moduli stabilization, it has been shown that e.g. compactifications of type IIB (on Calabi-Yau orientifolds) with non-trivial backgrounds of NSNS and RR 3-form field strength fluxes [12] generically fix the vevs of the dilaton, and all complex structure moduli $[13,14,15,16,17,19,20,18]$. Thus the status concerning dilaton/moduli stabilization (in these and other compactifications with fluxes) has substantially improved. Unfortunately, no similar progress has been achieved concerning our understanding of the cosmological constant, which is still assumed to be tuned (in a discrete manner, e.g. by choices of flux quanta) to a small value.

In the present article we address the question of the structure of SUSY-breaking soft terms in Type IIB models with the charged fields living on the volume of D3-branes. The idea is to explore the structure of these soft terms, in an as model-independent way as possible. We consider D3-branes located at a smooth (or orbifold) point in compact space, in a quite general closed string background. The non-trivial background (for instance, the presence of NSNS and RR 3-form fluxes) gives rise in general to SUSYbreaking soft terms on the D3-brane worldvolume theory, which can be computed by coupling the closed string supergravity background to the D3-brane worldvolume theory using the Dirac-Born-Infeld (DBI) and Chern-Simons (CS) D3-brane action 2. It is important to emphasize that, in order to obtain the soft terms, only local information of the background around the position of the branes is required. Thus we will perform an expansion of the closed string background around the positions of the branes, and obtain the corresponding expansion of soft terms. Namely, to lowest order one generically (unless the D3-branes happen to sit at a singularity) gets the $\mathcal{N}=4$ super Yang-Mills action. Subsequent terms in the Taylor expansion of the supergravity background induce new operators on the worldvolume field theory, which break softly the $\mathcal{N}=4$ supersymmetry. The same technique may be applied to the case of the D3-branes sitting at an orbifold singularity, where the gauge sector may contain chiral fermions (and be phenomenologically interesting), and the background breaks softly the $\mathcal{N}=1$ supersymmetry of the worldvolume theory.

The structure of these soft terms depends on the structure of the underlying closed string background. For instance, they depend on the tensor components of the background 3-form flux $G_{i j k}$. We find that in some cases one obtains soft terms of the kind

\footnotetext{
${ }^{2}$ This approach was already applied to a particular orbifold example in [21].
} 
already found in the past in heterotic compactifications. Thus, as remarked in [14], an imaginary self-dual (ISD) 3-form flux leads to no soft terms, corresponding to the no-scale [22] (or modulus dominance) models. On the other hand we find that an imaginary anti self-dual (IASD) $(3,0)$ background flux gives rise to soft terms corresponding to the 'dilaton domination' of old $[2,3,4]$. More general tensor structures for $G_{i j k}$ give rise to more general possibilities. The method also applies in backgrounds with other sources for SUSY-breaking, for instance it allows to compute the soft terms induced on the D3-branes from other distant sources like e.g. anti-D3-branes and orientifold O3-planes. In these examples, which involve no 3-form flux, only scalar masses (no gaugino masses) are induced.

The D3-brane system in a local closed string background may be embedded into a complete compactification, so the structure of soft terms that we obtain may be present in a variety of Type IIB compactifications. In particular, the system may be embedded in a Calabi-Yau orientifold or F-theory compactification, such as those considered in [14]. This provides an alternative derivation of the soft terms, by using the $\mathcal{N}=1$ supergravity formalism, which allows to identify some of the particular background data (like the 3-form fluxes) with auxiliary components of certain supermultiplets (like the dilaton, and Kähler and complex structure moduli). This procedure makes contact with the one advocated in $[2,3,4]$ for the heterotic compactifications. However, in the present Type IIB analysis there is an explicit origin for SUSY-breaking, which is the underlying supergravity background which includes 3 -form fluxes.

We also observe that the $\mathcal{N}=1$ supergravity effective action, with a superpotential induced by $G_{i j k}$ fluxes, is invariant under the $S L(2, \mathbf{Z})$ S-duality for the complex dilaton [23] directly inherited from Type IIB S-duality. The corresponding soft terms are thus also S-duality invariant.

As we remark in the main text, the soft terms corresponding to dilaton domination have remarkable finiteness properties [24, 25, 26]. Indeed, an $\mathcal{N}=4$ theory with the addition of this class of soft terms remains perturbatively finite [27, 25, 28, 29]. The reason for this fact was never very clear when this observation was made. In the present context we show that the finiteness properties are a consequence of holography and the fact that the IASD $(3,0) G_{i j k}$ background has a constant dilaton.

As we have emphasized, the method presented to compute soft terms may be applied to explicit compact models. A large class of type IIB Calabi-Yau or F-theory compactifications was introduced in [13, 14], with O3-planes, D3-branes and ISD fluxes. In that class of models the fluxes are ISD, and soft terms are absent on the worldvolume 
of D3-branes due to the no-scale structure. On the other hand we point out that locating $\overline{\mathrm{D} 3}$ 's on such ISD backgrounds does give rise to interesting SUSY-breaking soft terms on the worldvolume of the $\overline{\mathrm{D} 3}$ 's. As an application of these ideas we show an specific example based on a $\mathbf{Z}_{\mathbf{3}}$ Type IIB orientifold model with $\overline{\mathrm{D} 3}-$, D7-branes and antibranes. The set of branes at the $\mathbf{Z}_{\mathbf{3}}$ singularity gives rise to a realistic left-right symmetric $S U(3) \times S U(2)_{L} \times S U(2)_{R} \times U(1)_{B-L}$ three generation model. The underlying ISD flux gives rise to soft terms of the dilaton dominated type on the worldvolume of the $\overline{\mathrm{D} 3}$-branes and at the same time helps in cancelling RR-tadpoles.

Another interesting application concerns the structure of mass scales in the theory. In particular, one would like to identify the soft terms with those required by the MSSM for its viability. We find that soft masses are of order $M_{s}^{2} / M_{p}$, with $M_{s}$ the string scale. In order to obtain soft terms of order the electroweak scale one thus needs to have $M_{s}=10^{10} \mathrm{GeV}$. As described in the text, this is compatible with the observed value of the Planck scale if the compactification radius is three orders of magnitude smaller than the string scale. Alternatively, if some large warp factor is present, the smallness of the weak scale could be due to the warping and still have $M_{s}$ of order the Planck scale.

The structure of the present paper is as follows. In section 2 we describe the computation of the general soft terms by expanding the D3-brane worldvolume action in a general supergravity background. In section 3 we compute the soft terms on a D3-brane in a general background of 3-form fluxes. Several important classes of flux backgrounds, and the corresponding soft terms are discussed in section 3.1. In section 4 we discuss soft terms arising from supersymmetry breaking by distant antiD3-branes. In section 5 we comment on the different pattern of soft terms expected on the worldvolume of D7-branes. In section 6 we show how to describe soft terms for D3-branes at orbifold singularities, in particular in examples leading to a chiral worldvolume spectrum, and give some examples. In section 7 we describe the effect of flux-induced SUSY-breaking from the viewpoint of the effective $4 \mathrm{~d} \mathcal{N}=1$ supergravity action, and identify the auxiliary fields acquiring vevs with different components of the background flux. We also discuss some interesting properties of soft terms, like their S-duality invariance, and the relation between finiteness and dilaton domination via holography. In section 8 we apply our results to the computation of soft terms in a semirealistic 3-family left-right symmetric extension of the Standard Model. Some phenomenological implications of our results are described in section 9, and we leave some conclusions and comments for section 10. 


\section{SUSY-breaking soft terms and fluxes in non-compact spaces}

In this section we describe the computation of soft terms ${ }^{3}$ from the perspective of the worldvolume field theory on a D3-brane in a quite general IIB supergravity background. To do that we Taylor expand the supergravity background around the location of the branes. The transverse coordinates correspond to the worldvolume scalars of the branes. Thus the first orders in this expansion will yield the lowest dimensional terms we are interested on.

\section{$2.1 \quad$ General strategy}

In a generic situation, the dynamical fields on the worldvolume of a stack of $N$ D3branes are $U(N)$ gauge bosons, four adjoint Majorana fermions $\psi^{a}$, and six real adjoint scalars $\phi^{m}$.

In principle we should take the D3-brane action, given by a Dirac-Born-Infeld (DBI) term and a Chern-Simons (CS) term. Our conventions are described in appendix C. Following [30] the Dirac-Born-Infeld piece ${ }^{4}$, is given by

$S_{D 3}=-\mu_{3} \int d^{4} x \operatorname{Tr}\left(e^{-\phi} \sqrt{-\operatorname{det}\left(P\left[E_{\mu \nu}+E_{\mu m}\left(Q^{-1}-\delta\right)^{m n} E_{n \nu}\right]+\sigma F_{\mu \nu}\right) \operatorname{det}(Q)}\right)$

where $P[M]$ denotes the pullback of the 10d background $M$ onto the D3-brane worldvolume, and

$$
\begin{aligned}
E_{M N} & =G_{M N}-B_{M N} \\
Q_{n}^{m} & =\delta^{m}{ }_{n}+i \sigma\left[\phi^{m}, \phi^{p}\right] E_{p n} \\
\sigma & =2 \pi \alpha^{\prime}
\end{aligned}
$$

The Chern-Simons piece is given by

$$
S_{C S}=\mu_{3} \int \operatorname{Tr}\left(P\left[e^{i \sigma \mathbf{i}_{\phi} \mathbf{i}_{\phi}}\left(\sum_{n} C^{(n)}+\frac{1}{2} B_{2} \wedge C_{2}\right) e^{-B}\right] e^{\sigma F}\right) .
$$

where $i_{\phi} C_{(p)}$ denotes contraction of a leg of the $p$-form, transverse to the D3-brane, with the associated worldvolume scalar [30]. The above action is the bosonic sector

\footnotetext{
${ }^{3}$ See appendix A for a short review of $\mathcal{N}=1$ SUSY-breaking soft terms and their ultraviolet properties.

${ }^{4}$ There is the familiar question of the non-abelian extension of the DBI action. For the soft terms to be discussed in the present paper, only the leading $\alpha^{\prime}$ order terms are relevant, for which no ambiguity arises.
} 
of the full supersymmetric action. The relevant piece of the fermionic sector will be discussed later on.

The relative sign between the DBI and CS terms corresponds to the relative sign between the tension and charge of the 3-brane, and distinguishes D3-branes and antiD3-branes (denoted $\overline{\mathrm{D} 3}$-branes in what follows).

The above action reduces at low energies (i.e. at leading order in $\alpha^{\prime}$ ) to the action of $\mathcal{N}=4$ super Yang-Mills (plus a constant piece corresponding to the tension). In particular the DBI piece gives rise to the gauge field and scalar kinetic terms, as well as the familiar commutator squared potential for the scalars, as we see below. The axion coupling from the CS terms provides the $\theta$ term of Super-Yang-Mills (SYM).

Notice that the familiar $S O(6)$ R-symmetry of $\mathcal{N}=4$ SYM (under which the worldvolume scalars and fermions transform in the representations $\mathbf{6}, \mathbf{4}$, respectively) is also present in the full D3-brane action. In fact, it corresponds to the geometric rotational symmetry in the space transverse to the D3-brane. As such, it is a local symmetry of the configuration, which constrains the possible coupling of D3-brane worldvolume fields with the 10d supergravity fields.

It is important to recall the interpretation of worldvolume scalars as coordinates in transverse space, via

$$
x^{m}=2 \pi \alpha^{\prime} \phi^{m} .
$$

This implies that for the 10d supergravity fields in the above action, the dependence on the coordinates $x^{m}, m=4, \ldots, 9$ is translated, from the viewpoint of the D3-brane worldvolume, into a dependence on the worldvolume scalars. This leads to a nice interpretation, as follows. One can expand the supergravity background around the trivial configuration (flat metric, constant dilaton, vanishing NSNS and RR background) as a power series in $x^{m}$. This is clearly an expansion around the location of the D3-branes, so that the lowest terms provide a good description of the local background felt by the D3-branes. There is also a corresponding expansion on the D3-brane worldvolume. Regarding the 10d background fields as expansions in powers of $\phi^{m}$, one can expand the action (2.1), (2.3) around the $\mathcal{N}=4$ SYM theory, finding an infinite set of terms, of higher and higher dimensions, deforming the original $\mathcal{N}=4$ theory. The $\alpha^{\prime}$ expansion in the D3-brane worldvolume, therefore corresponds to an expansion around the local geometry near the D3-branes. The truncation of the D3-brane effective field theory to operators of some dimension corresponds to using a local description of the supergravity background on which the D3-branes are embedded, to some order in the power expansion. 
From any of these two physical points of view, we are interested in the first few such terms. From the D3-brane worldvolume viewpoint, this corresponds to keeping the most relevant terms; we will restrict to terms of dimension at most 3 in the computation below, although more terms can be obtained using this technique. From the viewpoint of the $10 \mathrm{~d}$ geometry, this corresponds to taking a local description of the configuration. This is interesting since it is in general easier to analyze the behaviour of local geometries, and later on embed them in global compactifications. In this paper we center on quite general configurations of supergravity fields for D3-branes at a smooth point in non-compact Calabi-Yau threefolds (which locally correspond to D3-branes in flat space, or rather, flat except for the backreaction of the fluxes on the metric). We also discuss the case of D3-branes sitting at orbifold singularities, which lead to chiral theories and could have phenomenological relevance. These classes provide local models for a large set of IIB string compactifications. Finally, another important application of local configurations of D3-branes is the AdS/CFT correspondence, since only the local geometry around the D3-branes survives in the near-horizon limit. In this language, the computation of soft terms for a given background corresponds to the computation of the mapping between low-dimension operators in the gauge theory and the supergravity fields in its gravity dual.

An important comment is in order. In our computation we expand around a configuration of D3-branes. It is possible that the induced soft terms lead to some instability of this original configuration. One prototypical example is the appearance of linear terms (see below), or of instabilities triggered by scalar trilinear terms, which induce the appearance of non-commuting scalar vevs, and hence the polarization of the D3branes into spherical 5-branes [30, 31]. In such unstable situations, our computation is useful in that it shows the instability, but one would need to carry out the analogous expansion around the stable vacuum to correctly describe the physical system. However in many situations, as we will see later, there are scalar mass terms which either remove the polarized D3-brane minimum, or create a potential barrier to reach it, thus rendering the original configuration at least metastable. Thus our analysis applies without subtleties to these situations.

\subsection{The soft terms}

In this paper we will center on quite general backgrounds which preserve $4 \mathrm{~d}$ Poincare invariance, and include objects like D3-branes, antibranes, and 3-form fluxes in the transverse space. This implies some restriction, for instance we will not allow for e.g. 
a constant term in the expansion of the 5-form (i.e. we do not describe 5-form fluxes, but include 5-form backgrounds generated by sources). We will however find that this class of background has a lot of interesting physics. The supergravity backgrounds we consider follow the general ansatz

$$
\begin{aligned}
d s^{2} & =Z_{1}\left(x^{m}\right)^{-1 / 2} \eta_{\mu \nu} d x^{\mu} d x^{\nu}+Z_{2}\left(x^{m}\right)^{1 / 2} d s_{C Y}^{2} \\
\tau & =\tau\left(x^{m}\right) \\
G_{3} & =\frac{1}{3 !} G_{l m n}\left(x^{m}\right) d x^{l} d x^{m} d x^{n} \\
\chi_{4} & =\chi\left(x^{m}\right) d x^{0} d x^{1} d x^{2} d x^{3} \\
F_{5} & =d \chi_{4}+*_{10} d \chi_{4}
\end{aligned}
$$

where $G_{3}=F_{3}-\tau H_{3}$ (with $F_{3}\left(H_{3}\right)$ being the RR(NSNS) flux), and $d s^{2}{ }_{C Y}$ denotes the metric in transverse space, in the absence of flux backreaction, i.e. the Ricci-flat metric of the underlying Calabi-Yau. Since we are interested in a local description, it is enough for our purposes to take it to be flat $6 \mathrm{~d}$ metric.

In general, the above fields will be subject to the supergravity equations of motion, with sources. For the moment we do not impose the equations of motion, since they will depend on the particular sources in the configuration.

The fields in general will have a power expansion

$$
\begin{aligned}
Z_{1}^{-1 / 2} & =1+\frac{1}{2} K_{m n} x^{m} x^{n}+\ldots \\
Z_{2}^{1 / 2} & =1+\ldots \\
\tau & =\tau_{0}+\frac{1}{2} \tau_{m n} x^{m} x^{n} \\
\chi_{4} & =\left(\text { const. }+\frac{1}{2} \chi_{m n} x^{m} x^{n}+\ldots\right) d x^{0} d x^{1} d x^{2} d x^{3} \\
G_{l m n}\left(x^{m}\right) & =G_{l m n}+\ldots
\end{aligned}
$$

where the coefficients $K, F, G, \tau_{0}$ in the right hand side are constant, independent of $x^{m}$. The piece of the 5 -form background relevant for our purposes below can be given also as

$$
F_{5}=\frac{1}{2}\left(\chi_{m n}+\chi_{n m}\right) x^{m} d x^{n} d x^{0} d x^{1} d x^{2} d x^{3}+\ldots
$$

The order of expansion will be enough for our purposes, but it should be clear that the techniques below can be similarly applied to obtain higher-dimensional terms. The above expansion and the corresponding expansion in the D3-brane action are a generalization of that studied in [21] for D3-branes at an orbifold singularity. We will discuss 
the orbifold situation in section 6 , and see that it is a simple projection of our more general result.

A comment is in order. In general, the above expansions for e.g. the metric or dilaton may include terms linear in the coordinates. Following computations similar to the ones below, they in general lead to worldvolume field theory terms linear in the scalar fields $\phi^{m}$. This implies (unless a fine-tuning of terms of different origin occurs, so that the linear terms cancel) that the D3-branes are not sitting at an extremum of the worldvolume scalar potential, and therefore that the latter acquire vevs and run down the potential. Geometrically, the D3-branes are not sitting at a stable location, and tend to move and fall into e.g. gravitational potential wells. In appendix B.1 we discuss an explicit example of this situation, where a D3-brane in the supergravity background of a stack of anti D3-branes falls towards them. From the viewpoint of the worldvolume theory, the background induces soft terms including linear terms in the scalars, which forces them to acquire vevs. Since this kind of situation is unstable, in the above expression we assumed just terms that lead to scalar potentials starting at quadratic order.

In what follows we describe the lowest order terms arising from the above expansion in the action (2.1), (2.3). Starting with the DBI action, we obtain the following expansion for the different terms (notice that $E_{\mu n}=0$ in our ansatz)

$$
\begin{aligned}
\operatorname{Im} \tau & =\operatorname{Im} \tau_{0}+\frac{\sigma^{2}}{2} \operatorname{Im} \tau_{m n} \phi^{m} \phi^{n} \\
\operatorname{det}(Q)^{1 / 2} & =1-\frac{i \sigma}{2} B_{m n}\left[\phi^{n}, \phi^{m}\right]-\frac{\sigma^{2}}{4}\left[\phi^{m}, \phi^{n}\right]\left[\phi_{m}, \phi_{n}\right] \\
{\left[-\operatorname{det}\left(P\left[E_{\mu \nu}\right]\right)\right]^{1 / 2} } & =1-\frac{\sigma^{2}}{2} \partial_{\mu} \phi^{m} \partial^{\mu} \phi^{m}+\sigma^{2} K_{m n} \phi^{m} \phi^{n}
\end{aligned}
$$

Grouping things together, we obtain

$$
\begin{aligned}
\mathcal{L}_{\mathrm{DBI}}= & \frac{\mu_{3} \sigma^{2}}{g_{s}} \operatorname{Tr}\left[\frac{1}{2} \partial_{\mu} \phi^{m} \partial^{\mu} \phi^{m}-\frac{1}{4}\left[\phi^{m}, \phi^{n}\right]\left[\phi_{n}, \phi_{m}\right]-\right. \\
& \left.-\left(K_{m n}+\frac{g_{s}}{2} \operatorname{Im} \tau_{m n}\right) \phi^{m} \phi^{n}-\frac{1}{12} g_{s}\left(G_{3}-\bar{G}_{3}\right)_{l m n} \phi^{l}\left[\phi^{n}, \phi^{m}\right]\right]
\end{aligned}
$$

where we have used that $B_{m n}=\frac{1}{3} H_{l m n} x^{l}=\frac{1}{3} \sigma H_{l m n} \phi^{l}=\frac{1}{6} \sigma i g_{s}\left(G_{3}-\bar{G}_{3}\right)_{l m n} \phi^{l}$. Eq. (2.9) corresponds to the familiar bosonic lagrangian of $\mathcal{N}=4 \mathrm{SYM}$, in the first line, and some soft terms in the second.

It is clear that in general, the expansion of the background, and its corresponding expansion of the D3-brane action, can contain terms leading to additional cubic and quartic couplings in the scalars. About the former, they will be absent in our examples 
below, essentially due to symmetries of the background ansatz. About the latter, we ignore them since we center on at most dimension 3 soft terms. In any case, these terms can be straightforwardly introduced in the general expressions, as the interested reader may check.

Carrying out a similar expansion in the CS piece, we have several terms beyond the familiar $\mathcal{N}=4$ D3-brane CS action, involving

$$
\begin{aligned}
\left(i_{\phi} i_{\phi} C_{6}\right)_{0123} & =\frac{\sigma}{2}\left(C_{6}\right)_{0123 m n}\left[\phi^{n}, \phi^{m}\right] \\
\left(C_{4}\right)_{0123} & =\frac{\sigma^{2}}{2} \chi_{m n} \phi^{m} \phi^{n}
\end{aligned}
$$

Using that in our conventions $C_{6}$ is defined [31] through (C.12)

$$
-*_{10}\left(F_{3}-C H_{3}\right)=d C_{6}-H_{3} \wedge\left(C_{4}+\frac{1}{2} B_{2} \wedge C_{2}\right)
$$

and the fact that at leading order in the scalars $C_{4}$ is trivial, we have $\left(d C_{6}\right)_{0123 m n p}=$ $-\frac{1}{2}\left[*_{6}\left(G_{3}+\bar{G}_{3}\right)\right]_{m n p}$, and $\left(C_{6}\right)_{0123 m n}=-\frac{\sigma}{6}\left[*_{6}\left(G_{3}+\bar{G}_{3}\right)\right]_{m n p} \phi^{p}$.

The CS piece of the action hence reads

$$
\mathcal{L}_{C S}=\mu_{3} \sigma^{2} \operatorname{Tr}\left[\frac{1}{2}(\operatorname{Re} \tau) F_{\mu \nu} \widetilde{F}^{\mu \nu}+\frac{1}{2} \chi_{m n} \phi^{m} \phi^{n}-\frac{i}{12} *_{6}\left(G_{3}+\bar{G}_{3}\right)_{l m n} \phi^{l}\left[\phi^{n}, \phi^{m}\right]\right]
$$

Finally we should also carry out a similar expansion in the fermionic piece of the action to get the soft fermion masses (the only fermionic soft terms of dimension up to three). This can be obtained by dimensional reduction of the fermionic completion of the 10d supersymmetric DBI-CS action [32]. This expansion in powers of the fermionic fields has been worked out by different methods (see for example [33],[34]). In particular, here we are interested in the piece giving rise to fermionic masses and kinetic terms

$$
\mathcal{L}_{\text {ferm. }}=\frac{\sigma^{2} \mu_{3}}{g_{s}}\left(-\frac{1}{2} \bar{\Theta} \Gamma^{\mu} D_{\mu} \Theta+\frac{1}{48} g_{s} \bar{\Theta} \Gamma^{p q r} \Theta R e\left[(a+i b)^{2}\left(*_{6} G-i G\right)_{p q r}\right]\right)
$$

Here the kinetic terms indices are $4 \mathrm{~d}$, while the indices $p, q, r$ are $10 \mathrm{~d}$ indices, but running only over the directions transverse to the D3-branes, since only on these there is non-trivial flux background. Also $\Theta$ is the ten-dimensional Majorana-Weyl spinor formed by the massless Ramond open string states, and $a$ and $b$ fix the embedding of the D3-brane supersymmetry in the $10 \mathrm{~d} \mathcal{N}=2$ IIB supersymmetry by means of $\theta_{1}=a \sigma \Theta, \theta_{2}=b \sigma \Theta$, where $\theta_{1}, \theta_{2}$, are the two Majorana-Weyl spacetime spinors of $\mathcal{N}=2$ supergravity and $a^{2}+b^{2}=1$. We work in the choice $(a, b)=(1,0)$. 
Performing the reduction to four dimensions according to

$$
\Gamma^{\mu}=\gamma^{\mu} \otimes 1 \quad ; \quad \Gamma^{m}=\gamma_{(5)} \otimes \gamma^{m}
$$

we get the soft term fermionic lagrangian:

$\mathcal{L}_{\text {ferm. }}=\frac{\mu_{3} \sigma^{2}}{g_{s}}\left(-\frac{1}{2} \sum_{a} \bar{\psi}^{a} \gamma^{\mu} D_{\mu} \psi^{a}+\frac{g_{s}}{96}\left(*_{6} G_{3}-i G_{3}\right)_{m n p} \sum_{a} \psi^{a}\left(\gamma^{m n p}\right)_{a b} \psi^{b}+\right.$ h.c. $)$

Here the Dirac matrices act on the $S O(6)$ weight space corresponding to the four different Majorana fermions on the D3-brane, labeled by $a, b, \ldots$. Notice that there is no Dirac matrix acting on the $4 \mathrm{~d}$ spacetime spinor indices, so this is indeed a mass term. The Yukawa couplings of the $\mathcal{N}=4$ theory can be obtained by considering higher-dimensional terms in the expansion.

Notice that the result can be easily guessed, since it is the only possible $S O(6)$ invariant mass term. Namely, it contracts the imaginary anti self-dual part of the flux (which transforms in the representation 10 of $S O(6)$ ) with the symmetric product of two fermions in the $\overline{\mathbf{4}}$ (which transforms in the $\overline{\mathbf{1 0}}$ of $S O(6)$ ), see below.

In total, we have the soft term lagrangian, in the string frame,

$$
\begin{aligned}
\mathcal{L}_{\text {soft }} & =\frac{\mu_{3} \sigma^{2}}{g_{s}} \operatorname{Tr}\left[-\left(K_{m n}-\frac{g_{s}}{2} \chi_{m n}+\frac{g_{s}}{2} \operatorname{Im} \tau_{m n}\right) \phi^{m} \phi^{n}+\right. \\
& \left.+\frac{i g_{s}}{6}\left(*_{6} G_{3}-i G_{3}\right)_{l m n} \phi^{l} \phi^{m} \phi^{n}+\text { h.c. }+\frac{g_{s}}{96}\left(*_{6} G_{3}-i G_{3}\right)_{l m n} \psi \gamma^{l m n} \psi+\text { h.c. }\right]
\end{aligned}
$$

This may be rewritten in the Einstein frame by means of $G_{E}=g_{s}{ }^{-1 / 2} G_{s t r}$ and $\gamma_{E}^{M}=$ $g_{s}{ }^{1 / 4} \gamma_{s t r}^{M}$. Rescaling all the fields in order to get rid of the $\mu_{3} \sigma^{2}=\frac{1}{2 \pi}$ global factor and the fermions in order to get the usual factor $i$ in the kinetic term, it takes the form

$$
\begin{aligned}
\mathcal{L}_{\mathrm{soft}}= & \operatorname{Tr}\left[-\left(K_{m n}-\frac{1}{2} \chi_{m n}+\frac{g_{s}}{2} \operatorname{Im} \tau_{m n}\right) \phi^{m} \phi^{n}+\right. \\
& \left.+\frac{i g_{s} \sqrt{2 \pi}}{6}\left(*_{6} G_{3}-i G_{3}\right)_{l m n} \phi^{l} \phi^{m} \phi^{n}+\text { h.c. }+\frac{i g_{s}^{1 / 2}}{96}\left(*_{6} G_{3}-i G_{3}\right)_{l m n} \psi \gamma^{l m n} \psi+\text { h.c. }\right]
\end{aligned}
$$

This is the main result of this section, the soft term lagrangian for a stack of D3-branes in a (quite) general supergravity background, of the form (2.6).

For D3-branes in Calabi-Yau manifolds, there is a preferred complex structure, associated to the $\mathcal{N}=1$ supersymmetry unbroken by the Calabi-Yau and the D3-brane. Namely, the complex structure in which the $S U(3)$ invariant spinor satisfies $\gamma^{\overline{1}} \xi=0$. It is useful to combine the D3-brane worldvolume fields in a vector multiplet and three chiral multiplets under this $\mathcal{N}=1$ supersymmetry, and express the soft terms in 
complex notation. This also simplifies the comparison with the familiar supersymmetry breaking terms in $\mathcal{N}=1$ supersymmetric field theories.

For concreteness, take $z^{1}=\frac{1}{\sqrt{2}}\left(x^{4}+i x^{5}\right), z^{2}=\frac{1}{\sqrt{2}}\left(x^{6}+i x^{7}\right), z^{3}=\frac{1}{\sqrt{2}}\left(x^{8}+i x^{9}\right)$, and introduce the complex scalars $\Phi^{1}=\frac{1}{\sqrt{2}}\left(\phi^{4}+i \phi^{5}\right), \Phi^{2}=\frac{1}{\sqrt{2}}\left(\phi^{6}+i \phi^{7}\right), \Phi^{3}=\frac{1}{\sqrt{2}}\left(\phi^{8}+i \phi^{9}\right)$. We also use $\Phi^{\overline{1}}$ to denote $\Phi_{i}^{*}$. Denoting the four-plet of fermions by their $S O(6)$ weights, the fermion $\frac{1}{2}(+++)$ belongs to the vector multiplet, and is referred to as the gaugino, denoted $\lambda$. The fermions combining with the above complex scalars to give chiral multiples are $\Psi^{1}, \Psi^{2}, \Psi^{3}$, corresponding to the weights $\frac{1}{2}(+--), \frac{1}{2}(-+-)$ and $\frac{1}{2}(--+)$, respectively.

In a preferred complex structure, only an $S U(3)$ (times $U(1)$ ) subgroup of the $S O(6)$ symmetry is manifest. The antisymmetric flux $G_{m n p}$ transforms as a 20-dimensional reducible $S O(6)$ representation, decomposing as $20=\overline{10}+10$. The irreducible representations $\overline{10}, 10$ correspond to the imaginary self-dual (ISD) $G_{(3)}^{+}$and imaginary anti self-dual (IASD) $G_{(3)}^{-}$parts, respectively, defined as

$$
G_{(3)}^{ \pm}=\frac{1}{2}\left(G_{(3)} \mp i *_{6} G_{(3)}\right) \quad ; \quad *_{6} G_{(3)}^{ \pm}= \pm i G_{(3)}^{ \pm}
$$

It is useful to classify the components of the ISD and IASD parts of $G_{3}$ according to their behaviour under $S U(3), 10=6+3+1$. For that purpose, we introduce the tensors [35]

$$
\begin{aligned}
S_{i j} & =\frac{1}{2}\left(\epsilon_{i k l} G_{j \bar{k} \bar{l}}+\epsilon_{j k l} G_{i \bar{k} \bar{l}}\right) \\
A_{\bar{i} \bar{j}} & =\frac{1}{2}\left(\epsilon_{\bar{i} \bar{k} \bar{l}} G_{k l \bar{j}}-\epsilon_{\bar{j} \bar{k} \bar{l}} G_{k l \bar{i}}\right)
\end{aligned}
$$

defined in terms of the complex components of $G_{3}$, and which transform in the representation 6 and $\mathbf{3}$ under $S U(3)$. One similarly defines $S_{\overline{i \bar{j}}}$ and $A_{i j}$.

The ISD condition allows for a $(0,3)$ component, a $(2,1)$ primitive part ${ }^{5}$ which corresponds to $S_{\overline{i j}}$, and a $(1,2)$ non-primitive part corresponding to $A_{i j}$. The IASD condition allows for a $(3,0)$ part, a $(1,2)$ primitive part $\left(S_{i j}\right)$ and a $(2,1)$ non-primitive one $\left(A_{\bar{i} \bar{j}}\right)$. The corresponding $S U(3)$ representations are given in table 1 .

Using the complex notation, and these definitions, we have the (Einstein frame) soft term lagrangian

$$
\begin{aligned}
& \mathcal{L}=\operatorname{Tr}\left[-\left(2 K_{i \overline{\mathrm{j}}}-\chi_{i \overline{\mathrm{j}}}+g_{s}(\operatorname{Im} \tau)_{i \overline{\mathrm{j}}}\right) \Phi^{i} \Phi^{\overline{\mathrm{j}}}-\frac{1}{2}\left(2 K_{i j}-\chi_{i j}+g_{s}(\operatorname{Im} \tau)_{i j}\right) \Phi^{i} \Phi^{j}+\right.\text { h.c. } \\
& +g_{s} \sqrt{2 \pi}\left[\frac{1}{3} G_{123} \epsilon_{i j k} \Phi^{i} \Phi^{j} \Phi^{k}+\frac{1}{2} \epsilon_{i \bar{j} \bar{l}}\left(S_{l k}-\left(A_{\overline{l k}}\right)^{*}\right) \Phi^{\bar{i}} \Phi^{\bar{j}} \Phi^{k}+\text { h.c. }\right]+ \\
& \left.+\frac{g_{s}^{1 / 2}}{2 \sqrt{2}}\left[G_{123} \lambda \lambda+\frac{1}{2} \epsilon_{i j k} A_{\bar{j} \bar{k}} \Psi^{i} \lambda+\frac{1}{2} S_{i j} \Psi^{i} \Psi^{j}+\text { h.c. }\right]\right]
\end{aligned}
$$

\footnotetext{
${ }^{5} G_{3}$ is primitive if $G_{3} \wedge J=0$ with $\mathrm{J}$ the Kähler form.
} 


\begin{tabular}{|c|c|c||c|c|c|}
\hline & ISD & & & IASD & \\
\hline$S U(3)$ rep. & Form & Tensor & $S U(3)$ rep. & Form & Tensor \\
\hline \hline$\overline{1}$ & $(0,3)$ & $G_{\overline{1} \overline{2} \overline{3}}$ & 1 & $(3,0)$ & $G_{123}$ \\
\hline$\overline{6}$ & $(2,1)_{P}$ & $S_{\bar{i} \bar{j}}$ & 6 & $(1,2)_{P}$ & $S_{i j}$ \\
\hline$\overline{3}$ & $(1,2)_{N P}$ & $A_{i j}$ & 3 & $(2,1)_{N P}$ & $A_{\bar{i} \bar{j}}$ \\
\hline
\end{tabular}

Table 1: $S U(3)$ decomposition of antisymmetric $G_{(3)}$ fluxes.

where we have defined

$$
\begin{aligned}
(\operatorname{Im} \tau)_{i \overline{\mathrm{j}}} & =\frac{1}{2 i}\left(\tau_{i \overline{\mathrm{j}}}-\left(\tau_{j \bar{i}}\right)^{*}\right) \\
(\operatorname{Im} \tau)_{i j} & =\frac{1}{2 i}\left(\tau_{i j}-\left(\tau_{\overline{\mathrm{j}} \bar{i}}\right)^{*}\right)
\end{aligned}
$$

It is standard to write the soft terms using the couplings of the underlying $\mathcal{N}=1$ supersymmetric theory. In our case, one can write the $\mathcal{N}=1$ superpotential of the $\mathcal{N}=4$ theory as

$$
W_{\mathcal{N}=4}=g \frac{\sqrt{2}}{3} \epsilon_{i j k} \Phi^{i} \Phi^{j} \Phi^{k}=\frac{1}{3 !} h_{i j k} \Phi^{i} \Phi^{j} \Phi^{k}
$$

with $g=\sqrt{2 \pi g_{s}}$ the gauge coupling constant. Thus, in the notation of Appendix A, we have soft SUSY breaking terms as follows:

$$
\begin{aligned}
m_{i j}^{2} & =2 K_{i \overline{\mathrm{j}}}-\chi_{i \overline{\mathrm{\jmath}}}+g_{s}(\operatorname{Im} \tau)_{i \overline{\mathrm{j}}} \\
B_{i j} & =2 K_{i j}-\chi_{i j}+g_{s}(\operatorname{Im} \tau)_{i j} \\
A^{i j k} & =-h^{i j k} \frac{g_{s}^{1 / 2}}{\sqrt{2}} G_{123} \\
C^{i j k} & =+h^{i j l} \frac{g_{s}^{1 / 2}}{2 \sqrt{2}}\left(S_{l k}-\left(A_{\overline{l k}}\right)^{*}\right) \\
M^{a} & =\frac{g_{s}^{1 / 2}}{\sqrt{2}} G_{123} \\
\mu_{i j} & =-\frac{g_{s}^{1 / 2}}{2 \sqrt{2}} S_{i j} \\
M_{g}^{i a} & =\frac{g_{s}^{1 / 2}}{4 \sqrt{2}} \epsilon_{i j k} A_{\bar{j} \bar{k}}
\end{aligned}
$$

Several comments are in order. First note that all dimension 3 SUSY-breaking soft terms only depend on the three form flux, and not on the 5-form, warping or dilaton contributions. Note also that, even before applying supergravity equations of motion, there are certain relationships between the different soft terms. In particular $\mathcal{N}=1$ 
gaugino masses and $A^{i j k}$ trilinear terms are related by

$$
A^{i j k}=-h^{i j k} M^{a}
$$

Note also that in certain situations the dimension 3 soft terms are consistent with one unbroken $\mathcal{N}=1$ supersymmetry. In particular, if there is only $(1,2)$ IASD flux (i.e. $S_{i j} \neq 0$ ), one has the relationship

$$
C^{i j k}=-h^{i j l} \mu_{k l}
$$

which corresponds to the trilinear scalar term arising from an effective $\mathcal{N}=1$ superpotential obtained by adding a mass piece $\frac{1}{2} \mu_{i j} \Phi^{i} \Phi^{j}$ to the $\mathcal{N}=4$ superpotential. If in addition the scalars $\Phi^{i}$ and fermions $\Psi^{i}$ have equal masses the terms would preserve $\mathcal{N}=1$. We discuss these different situations, as well as additional relationships appearing after imposing locally the supergravity equations of motion below.

The result eq.(2.19) is more general than that in [21] in several respects. In particular we do not impose orbifold symmetries. Hence our above expression contains some interesting new terms, like for instance scalar mass terms of the form $\Phi^{i} \Phi^{j}$, commonly known as B-terms in the SUSY-breaking soft term literature, or non-holomorphic trilinear scalar terms. Although such terms are projected in e.g. abelian orbifold projections of odd order, they are important whenever the orbifold allows for invariant $(1,2)$ forms (like for example $\mathbf{C}^{\mathbf{3}} / \mathbf{Z}_{\mathbf{4}}$ ). This situation is briefly discussed in section 6 .

Let us mention that the above result changes if one considers anti-D3-branes instead of D3-branes, via a flip in the relative sign between the DBI and CS pieces of the action. This implies an additional sign in the pieces related to $\chi_{m n}$ and $*_{6} G_{3}$. This effectively exchanges the roles of the imaginary self-dual (ISD) and imaginary anti selfdual (IASD) parts of $G_{3}$, at the level of the soft term lagrangian. Hence the effect of ISD (resp. IASD fluxes) on D3-branes will be equivalent to the effect of IASD (resp. ISD) fluxes on $\overline{\mathrm{D} 3}$-branes. In other words, a simultaneous parity in the $4 \mathrm{~d}$ Poincare dimensions and the $6 \mathrm{~d}$ transverse coordinates (which is a symmetry of type IIB theory) exchanges the roles of ISD and IASD fluxes and turns D3-branes into $\overline{\mathrm{D} 3}$-branes, and vice versa.

An additional comment is in order. The parameters in the supergravity background are in general constrained by the supergravity equations of motion. The explicit relations in different examples depend on the corresponding sources. We have chosen not to impose these constraints in the above expressions, in order to be completely general. In coming discussions and examples, however, we will ensure that the background obeys 
the supergravity equations of motion. Then the metric, dilaton and 5-form background are related to the corresponding sources, which we usually take to be just the fluxes themselves, as in next section. The above expressions, however, would be valid if other kinds of sources are present, see for instance section 4.

\section{D3-branes in pure flux backgrounds and equa- tions of motion}

One very interesting general class of configurations are those where the (only) source of warping, dilaton and 5-form are 3-form fluxes.

The structure of the background is given by our above ansatz. As discussed, the supergravity equations of motion of course imply relations between the parameters in the ansatz for the metric, dilaton, and 5-form, and the 3-form fluxes that source them. In particular, we will find that equations of motion relate the form of mass squared terms to the 3 -form fluxes. The $D=10$ supergravity equations of motion (C.4-C.7) lead to the following results. From equation

$$
\nabla^{M}\left(\frac{i}{2} e^{\phi} \partial_{M} \tau\right)-\frac{1}{2} e^{2 \phi} \partial^{M} C \partial_{M} \tau=\frac{g_{s}}{24} G_{M N P} G^{M N P}
$$

to lowest order we get the constraint

$$
i \sum \tau_{l \bar{l}}=\frac{1}{2}\left(G_{123} G_{\overline{1} \overline{2} \overline{3}}+\frac{1}{4} S_{l k} S_{\overline{l k}}+\frac{1}{4} A_{l k} A_{\overline{l k}}\right)
$$

From

$$
\begin{aligned}
R_{M N}= & \frac{1}{4} e^{2 \phi} \partial_{M} \tau \partial_{N} \tau^{*}+\frac{1}{4} e^{2 \phi} \partial_{M} \tau^{*} \partial_{N} \tau+\frac{g_{s}^{2}}{96} F_{M Q R S T} F_{N}{ }^{Q R S T} \\
& +\frac{g_{s}}{8}\left(G_{M}{ }^{P Q} G_{N P Q}^{*}+G_{N}{ }^{P Q} G_{M P Q}^{*}-\frac{1}{6} g_{M N} G_{P Q R}^{*} G^{P Q R}\right)
\end{aligned}
$$

we obtain

$$
4 \sum K_{l \bar{l}}=\frac{g_{s}}{2}\left(\left|G_{123}\right|^{2}+\left|G_{\overline{1} \overline{2} \overline{3}}\right|^{2}+\frac{1}{4} \sum_{i j}\left(\left|S_{i j}\right|^{2}+\left|S_{\overline{i j}}\right|^{2}+\left|A_{i j}\right|^{2}+\left|A_{\overline{i j}}\right|^{2}\right)\right)
$$

Finally from

$$
d F_{5}=\frac{i g_{s}}{2} G_{3} \wedge \bar{G}_{3}
$$

we obtain the constraint

$$
-2 \sum \chi_{l \bar{l}}=\frac{g_{s}}{2}\left(\left|G_{123}\right|^{2}-\left|G_{\overline{1} \overline{2} \overline{3}}\right|^{2}+\frac{1}{4} \sum_{i j}\left(\left|S_{i j}\right|^{2}-\left|S_{\bar{i} \bar{j}}\right|^{2}-\left|A_{i j}\right|^{2}+\left|A_{\bar{i} \bar{j}}\right|^{2}\right)\right)
$$


Combining these results one finds the following constraint on the scalar mass matrix

$$
\begin{aligned}
m_{1}^{2}+m_{2}^{2}+m_{3}^{2}= & \frac{g_{s}}{2}\left[\left|G_{123}\right|^{2}+\frac{1}{4} \sum_{i j}\left(\left|S_{i j}\right|^{2}+\left|A_{\bar{i} \bar{j}}\right|^{2}\right)-\right. \\
& \left.-\operatorname{Re}\left(G_{123} G_{\overline{1} \overline{2} \overline{3}}+\frac{1}{4} S_{l k} S_{\overline{l k}}+\frac{1}{4} A_{l k} A_{\overline{l k}}\right)\right]
\end{aligned}
$$

The first three terms in the right hand side may be rewritten in terms of the trace of the masses $M_{i j}, i, j=0,1,2,3$ of the four fermions $\lambda$ and $\Psi_{1,2,3}$. One then has

$$
m_{1}^{2}+m_{2}^{2}+m_{3}^{2}=\operatorname{Tr}\left(M_{i j}\left(M^{i j}\right)^{*}\right)-\frac{g_{s}}{2} \operatorname{Re}\left(G_{123} G_{\overline{1} \overline{2} \overline{3}}+\frac{1}{4} S_{l k} S_{\overline{l k}}+\frac{1}{4} A_{l k} A_{\overline{l k}}\right)
$$

Notice that the supertrace of the masses of the scalars and spin $1 / 2$ fermions vanishes in certain cases. In particular it vanishes if all the ISD fluxes vanish (i.e. $G_{\overline{1} \overline{2} \overline{3}}=$ $\left.S_{\overline{l k}}=A_{l k}=0\right)$. It also vanish if each ISD(IASD) background is not present when its IASD(ISD) partner flux is present.

Notice that as observed in section IV.A of [31], the masses are not fully determined by the fluxes, only the trace of the mass squared matrix is. This is because the masses can be changed (keeping the trace fixed) by contributions from e.g. homogeneous vacuum solutions to the metric equation of motion. In appendix B.2 we describe one such situation. Since $B_{i j}$ terms do not contribute to the trace of the scalar masses, the local equations of motion do not give any constraint on them either.

In the case of the scalar mass terms $m_{i j}^{2}$ one can get a relationship with the fluxes if a definite choice of boundary conditions for the metric, 5-form and dilaton backgrounds is made (which fixes the ambiguity of the vacuum solution). For example, assuming spherically symmetric boundary conditions, which are natural if no other sources are present, the soft masses are all equal, with the squared mass equal to one third of the RHS in (3.7). Then one gets $m_{1}^{2}=m_{2}^{2}=m_{3}^{2}=m^{2}$ with

$$
m^{2}=\frac{g_{s}}{6}\left[\left|G_{123}\right|^{2}+\frac{1}{4} \sum_{i j}\left(\left|S_{i j}\right|^{2}+\left|A_{\overline{i j}}\right|^{2}\right)-\operatorname{Re}\left(G_{123} G_{\overline{1} \overline{2} \overline{3}}+\frac{1}{4} S_{l k} S_{\overline{l k}}+\frac{1}{4} A_{l k} A_{\overline{l k}}\right)\right]
$$

It is important to notice that in the presence of other sources not respecting the spherical symmetry, one in general generates additional mass terms which modify the scalar mass matrix (while preserving its trace).

\section{$3.1 \quad$ Examples}

In this section we describe some prototypical examples illustrating a variety of possible classes of models. Clearly, combinations of the diverse ingredients can lead to interesting results as well. 


\subsubsection{ISD fluxes and no-scale structure}

A familiar choice of flux configurations is to take the flux density $G_{3}$ to be imaginary self-dual, $*_{6} G_{3}=i G_{3}$. This choice is interesting since in $[13,14]$ it was found that compactification on CY orientifolds with fluxes, to $4 \mathrm{~d}$ Minkowski space, required the fluxes to satisfy this condition. In complex coordinates, the ISD condition allows for primitive $(2,1)$, non-primitive $(1,2)$ and $(0,3)$ components for $G_{3}$.

The explicit supergravity background describing ISD fluxes is of the form

$$
\begin{aligned}
d s^{2} & =Z^{-1 / 2} \eta_{\mu \nu} d x^{\mu} d x^{\nu}+Z^{1 / 2} d x^{m} d x^{m} \\
\tau & =\tau_{0} \\
G_{3} & =G_{l m n} d x^{l} d x^{m} d x^{n}=-i\left(*_{6} G\right)_{l m n} \\
\chi_{4} & =\frac{1}{Z} d x^{0} d x^{1} d x^{2} d x^{3} \\
F_{5} & =d \chi_{4}+*_{10} d \chi_{4}
\end{aligned}
$$

with $Z=1-\left(g_{s} / 72\right) G^{2} r^{2}$, with $G^{2}=G_{l m n}^{*} G^{l m n}$ and $r^{2}=\left(x^{4}\right)^{2}+\ldots\left(x^{9}\right)^{2}{ }^{6}$.

As is obvious from the previous discussion, pure ISD fluxes do not give rise to any SUSY-breaking soft terms. Still, equations of motion only fix the trace of the mass ${ }^{2}$ so that one has

$$
m_{1}^{2}+m_{2}^{2}+m_{3}^{2}=0
$$

If, as discussed above, one assumes spherically symmetric boundary conditions one gets

$$
m_{1}^{2}=m_{2}^{2}=m_{3}^{2}=0
$$

The absence of soft terms is a consequence of cancellations between the DBI and CS terms of the D3-brane action. Hence, they are consequence of the almost BPS nature of ISD fluxes [14], whose supergravity solution is of the black 3-brane form. Notice that this is not a consequence of supersymmetry, which is present only if the flux is primitive $(2,1)$ in some complex structure, while the imaginary self-duality condition in principle allows for additional components.

The absence of soft terms is in agreement with the structure of the effective $4 \mathrm{~d}$ supergravity of the compactifications in [14], which are of the no-scale type [22], at leading order in $\alpha^{\prime}$. This will be discussed in section 7 .

\footnotetext{
${ }^{6}$ Notice that we have chosen a spherically symmetric solution for the warp factor, etc, in order to fix the metric, dilaton and 5-form in terms of the fluxes.
} 


\subsubsection{IASD fluxes}

Other interesting examples are provided by D3-branes in an imaginary anti self-dual $G_{3}$ flux background, $*_{6} G_{3}=-i G_{3}$. As discussed above, the configuration of D3-branes in IASD flux is equivalent to the situation of $\overline{\mathrm{D} 3}$-branes in an ISD flux. Hence it provides the kind of soft terms on a $\overline{\mathrm{D} 3}$-brane embedded in a compactification of the kind considered in [14]. This is interesting, given the role played by such configurations in recent proposals to construct de Sitter vacua in string theory [20].

An explicit supergravity background with IASD flux is given by eq.(3.10), except for an additional sign in the 5 -form background, and the fact that $G_{3}$ is IASD. This implies that there are no cancelations in this case ${ }^{7}$. The IASD condition allows for $(3,0)$, primitive $(1,2)$ and non-primitive $(2,1)$ components for $G_{3}$. Let us study them in turn.

\section{a) $(3,0)$ fluxes and dilaton domination}

An interesting case is when the IASD flux has only $(3,0)$ component, i.e, only $G_{123}$ is non-vanishing. From eq.(2.22), (3.8), (3.9) one gets (assuming spherically symmetric boundary conditions for simplicity)

$$
m^{2}=\frac{g_{s}}{6}\left|G_{123}\right|^{2} ; M^{a}=\frac{g_{s}^{1 / 2}}{\sqrt{2}} G_{123} ; A^{i j k}=-h^{i j k} \frac{g_{s}^{1 / 2}}{\sqrt{2}} G_{123}
$$

with the rest of the soft terms vanishing. Note the relationships

$$
A^{i j k}=-h^{i j k} M_{a} ; m^{2}=\frac{1}{3}|M|^{2}
$$

This type of soft terms ${ }^{8}$ are remarkable in several respects and have appeared previously in the literature. Indeed they correspond to the dilaton domination form discussed in $[3,4]$. Namely, soft terms satisfying these relation appear in $\mathcal{N}=1$ supergravity theories with spontaneous supersymmetry breaking due to a vacuum expectation value for the auxiliary field in the dilaton multiplet. The relation with our present configuration will be explained in section 7 , where we show that in compactifications

\footnotetext{
${ }^{7}$ This has been discussed in [36] for the scalar trilinear couplings, which were argued to lead to D3-brane polarization for any IASD flux background. Our analysis corrects this point, by noticing that one in general also obtains non-trivial scalar masses, which prevent the scalars from acquiring vevs. Hence in general we obtain stable (or metastable) configurations of non-polarized D3-branes, even in the presence of IASD fluxes.

${ }^{8}$ This theory could be referred to as $\mathcal{N}=0^{*}$ theory, in parallelism to the $\mathcal{N}=1^{*}$ theory in [31].
} 
the $(3,0)$ component of the flux is indeed the auxiliary field of the dilaton (with respect to the supersymmetry unbroken by the D3-brane).

Another interesting property of this type of boundary conditions, noted a long time ago, is that their presence leads to a $\mathcal{N}=0$ theory which is finite in all orders in perturbation theory $[27,25]$. We will come to this interesting property in section (7).

\section{b) $(1,2)_{P}$ fluxes and 'susy' soft terms}

Let us now consider configurations of a pure primitive $(1,2)$ flux, which we parametrize in terms of the tensor $S_{i j}$. From (2.22), (3.8), one gets

$$
m_{1}^{2}+m_{2}^{2}+m_{3}^{2}=\frac{g_{s}}{8} \sum_{i j}\left|S_{i j}\right|^{2} ; C^{i j k}=-h^{i j l} \mu_{k l} ; \mu_{i j}=-\frac{g_{s}^{1 / 2}}{2 \sqrt{2}} S_{i j}
$$

with the rest of the terms vanishing. It is easy to convince oneself that these terms are consistent with an effective $\mathcal{N}=1$ SUSY theory corresponding to a superpotential

$$
W=W_{\mathcal{N}=4}+\frac{1}{2} \mu_{i j} \Phi^{i} \Phi^{j}
$$

Consider for example the case in which only $S_{11}$ is non-vanishing. The above results are consistent with an $\mathcal{N}=1$ theory with two massless chiral multiplets, $\Phi^{2,3}$, and one

massive multiplet $\Phi^{1}$ with mass $\frac{g_{s}^{1 / 2}}{2 \sqrt{2}} S_{11}$. It is also easy to check that fluxes with just one off-diagonal component $S_{i j}$ lead to terms consistent with an $\mathcal{N}=2 \mathrm{SUSY}$.

This type of fluxes are of particular interest since they give rise to masses for nonchiral fermions. Such terms are required for instance in order to obtain a ' $\mu$-term' for the Higgs multiplets in the MSSM.

The reason underlying the $\mathcal{N}=1$ structure of soft terms for this kind of fluxes is presumably their relation to the flux background in the Polchinski-Strassler construction [31]. Indeed, it is possible to complement the above primitive $(1,2)$ fluxes with a suitable combination of a $(0,3)$ component, and a non-primitive $(1,2)$ component, such that they obey the constraints (3.30) in [35]. These additional pieces do not modify the soft terms, because they are ISD components (so that they do not generate trilinear terms or fermion masses) and such that the 'interference term' in scalar masses (3.8) vanishes. The conditions in [35], satisfied by this completed flux, guarantee that the system of the D3-brane and the flux preserve an $\mathcal{N}=1$ supersymmetry (although it is not the same preserved by a pure D3-brane system) and that the deformation of the $\mathcal{N}=4$ theory is provided by a superpotential $W$ related to the original $(1,2)_{P}$ flux 
as obtained above by direct analysis, namely $S_{i j} \sim \partial_{i} \partial_{j} W^{9}$. It would be interesting to understand the relation to other supersymmetric flux configuration in the literature $[37]$.

\section{c) $(2,1)_{N P}$ fluxes}

This flux is parametrized in terms of $A_{\bar{i} \bar{j}}$. From eq.(2.22), (3.8) one gets

$$
m_{1}^{2}+m_{2}^{2}+m_{3}^{2}=\frac{g_{s}}{8} \sum_{i j}\left|A_{\overline{i j}}\right|^{2} ; C^{i j k}=-h^{i j l} \frac{g_{s}^{1 / 2}}{\sqrt{2}}\left(A_{\overline{l k}}\right)^{*} ; M_{g}^{i a}=\frac{g_{s}^{1 / 2}}{4 \sqrt{2}} \epsilon_{i j k} A_{\bar{j} \bar{k}}
$$

These soft terms break supersymmetry completely. In CY compactifications, such type of flux cannot be turned on (since the existence of non-primitive fluxes requires the existence of non-trivial 5-forms to support the non-vanishing $G_{3} \wedge J$ ), so this situation is less interesting.

\subsubsection{Mixed ISD and IASD fluxes}

It is interesting to consider more general situations, with fluxes not purely ISD or IASD. For dim=3 soft terms both ISD and IASD contributions add linearly. However, as observed from eq.(3.7), the contributions to scalar masses upon imposing equations of motion do not add linearly, there is an interference term proportional to the products of ISD and IASD contributions. In particular if one has

$$
G_{\overline{1} \overline{2} \overline{3}}=G_{123} ; S_{\bar{i} \bar{j}}=S_{i j} ; A_{i j}=A_{\bar{i} \bar{j}}
$$

one can check that the 5-form background vanishes and the scalar masses also do. More general situations are obtained for arbitrary ISD and IASD fluxes.

As a prototype class of models, we consider turning on a flux with $(3,0)$ and $(0,3)$ components, namely

$$
G_{3}=G_{0} d z_{1} d z_{2} d z_{3}+G_{0}^{\prime} d \bar{z}_{1} d \bar{z}_{2} d \bar{z}_{3}
$$

In this case the supergravity solution is not of the black 3-brane form, so we use our general ansatz (2.6) and impose the equations of motion (C.7) on the coefficients of

\footnotetext{
${ }^{9} \mathrm{An}$ important point is that in our analysis we expand the worldvolume theory of a D3-brane, whereas in [31] the supersymmetric ground state was shown to correspond to dielectric D3-branes. Hence both D3-brane systems are different (meta)stable configurations of the same underlying $\mathcal{N}=1$ supersymmetric lagrangian.
} 
(2.7). We obtain for the soft terms (and spherically symmetric boundary conditions)

$$
\begin{aligned}
m^{2} & =\frac{g_{s}}{6}\left(\left|G_{0}\right|^{2}-\operatorname{Re}\left(G_{0} G_{0}{ }^{\prime}\right)\right) \\
M^{a} & =\frac{g_{s}^{1 / 2}}{\sqrt{2}} G_{0} ; A^{i j k}=-h^{i j k} \frac{g_{s}^{1 / 2}}{\sqrt{2}} G_{0}
\end{aligned}
$$

Clearly, allowing for these more general fluxes one is able to reproduce a rich pattern of soft terms on the D3-brane worldvolume. In particular, without loss of generality one can parametrize, (assuming $G_{0} \neq 0$, which would just give rise to no soft terms)

$$
G_{123}=\left|G_{0}\right| e^{i \delta} ; G_{\overline{1} \overline{2} \overline{3}}=\tan \theta e^{i \beta}\left|G_{0}\right|
$$

with $\delta, \beta$ real parameters. In terms of these one obtains for the scalar masses

$$
\begin{aligned}
m^{2} & =\frac{|M|^{2}}{3}[1-\tan \theta \cos (\delta+\beta)] \\
A^{i j k} & =-M h^{i j k}
\end{aligned}
$$

Note that this more general situation alters only the relative size of the scalar masses, for which a variety of possibilities now appear. Consider for example real flux backgrounds $(\beta=\delta=0 \bmod 2 \pi)$. For $\tan \theta=0$ one recovers the dilaton dominated soft terms discussed in the previous section. For $\tan \theta=1$ one gets vanishing scalar masses but non-vanishing $A$ and $M$ parameters. This corresponds to the case discussed above with a vanishing 5 -form. Note that in this case the trilinear couplings will destabilize the branes since a non-vanishing vev for the scalars will be energetically preferred. Finally, for $\tan \theta \ll-1$ one gets $m^{2} \gg M^{2}$ and scalar masses are much greater than gaugino and trilinear terms.

The above expressions could be interesting for phenomenological SUSY analysis if applied to the more realistic theories (yielding an open string spectrum close to the MSSM) instead of a $\mathcal{N}=4$ theory. Some steps in this direction are taken in sections 6,8 .

\section{SUSY-breaking by distant antibranes}

Our general formalism to compute the SUSY-breaking soft terms is not restricted to configurations of fluxes. Rather, it allows the computation of soft terms in other nonsupersymmetric situations, even if no fluxes are present. In particular, it provides an interesting way to compute the effect of supersymmetry breaking due to a set of distant antibranes, and can also be applied to compactifications based on [38], e.g. with 
a visible sector of supersymmetric D-branes, and supersymmetry broken in a sector of antibranes. This is interesting since there exist models with semirealistic spectrum in this class $[6,7]$, so it is natural to explore the basic features of SUSY-breaking soft terms in this setup. Moreover, anti-D3-branes play a prominent role in recent descriptions of de Sitter vacua in string theory [20].

Some relevant observations of this approach are discussed in this section, and we leave a more systematic discussion for future work. For the purposes of a general discussion, we center on the soft terms induced on a D3-brane (at the origin in $\mathbf{R}^{\mathbf{6}}$ ) due to the background created by a stack of $M$ distant anti-D3-branes (at the point $\vec{x}_{0}$ in $\mathbf{R}^{\mathbf{6}}$ ). In order for the supergravity background at the D3-brane location to be trustable, one should be in the regime where the $\overline{\mathrm{D} 3}$-branes are distant. Luckily, this is also the interesting situation, where one is far from the tachyonic regime, and also the situation of interest in brane inflation discussions [39].

The background is given by

$$
\begin{aligned}
d s^{2} & =Z\left(x^{m}\right)^{-1 / 2} \eta_{\mu \nu} d x^{\mu} d x^{\nu}+Z\left(x^{m}\right)^{1 / 2} d x^{m} d x^{m} \\
\chi_{4} & =\frac{1}{Z} d x^{0} d x^{1} d x^{2} d x^{3} \\
F_{5} & =d \chi_{4}+* d \chi_{4} \\
Z & =1-\frac{1}{2 \pi^{2}} \frac{M}{\left|\vec{x}-\overrightarrow{x_{0}}\right|^{4}}
\end{aligned}
$$

and there is a vanishing NSNS and RR flux background.

The computation of some terms from the DBI action is described in appendix B. Clearly, the leading terms show the instability of the configuration, namely a linear term and tachyonic mass terms for the worldvolume scalars, signalling the tendency of the D3-branes to approach the anti-D3-brane stack. Such instabilities can be avoided in explicit examples (like branes stuck at singularities, see section (8)), which are more involved but presumably retain the other main features of this simpler configuration. Hence we proceed to discuss these aspects, ignoring the instability-related terms. Instead of entering the detailed discussion, we prefer to make some general comments on soft terms due to antibrane supersymmetry breaking.

Antibrane supergravity backgrounds contain basically a warp factor and a 5-form background, and do not introduce any 3-form fluxes. Therefore, at the order we have studied, the soft terms on D3-branes would be just scalar masses (and possibly trilinear terms, arising from the metric and 5-form). In sharp contrast with the above examples with fluxes, the present situation does not lead to fermion masses.

This is strongly suggestive that the supersymmetry breaking induced by antiD3- 
branes is a D-term breaking. Indeed this nicely dovetails with properties of D-branes on Calabi-Yau manifolds, where the tension (or vacuum energy) associated to B-type branes (namely, branes wrapped on holomorphic cycles; in our case of $\overline{\mathrm{D} 3}$-branes at a point in the Calabi-Yau) is controlled by Kähler parameters, which couple to D3-branes as D-terms ${ }^{10}$. It would be nice to derive this from a $4 \mathrm{~d}$ supergravity approach, as done for flux SUSY-breaking in section 7.

From the phenomenological viewpoint, the absence of gaugino masses is an important drawback for this kind of supersymmetry breaking. Concerning the scalar potential, it is difficult to make model-independent statements. However, a general statement is that, since the anti-D3-branes are distant, they give no additional sources at the D3-brane location and the relation (3.7) is unchanged (and similarly for the 5 -form and dilaton equations). Hence the addition of distant antibranes in general changes the scalar masses, but leaves the trace of the scalar mass matrix unchanged (see section B.2 for further discussion and an explicit example). Hence, in models where the only source of scalar masses are distant antibranes, at least some scalars are tachyonic ${ }^{11}$.

In conclusion, although very interesting from the theoretical viewpoint, this source of SUSY-breaking, taken by itself, would not be phenomenologically viable. However, if distant antibranes are present in a configuration with other structures, like 3-form fluxes, they may lead to interesting substructures in the pattern of soft terms, like deviations from universality in an otherwise dilaton-domination SUSY-breaking configuration. This departure from universality, if applied to a realistic model, lead in general to Flavour Changing Neutral Currents (FCNC). These are also very much constrained experimentally, so distant antibrane SUSY-breaking should again not be the dominant source in order to avoid further phenomenological problems. We discuss in certain detail a semirealistic example in section 8 .

\section{$5 \quad$ D7-branes and fluxes}

It is interesting to point out that the absence of worldvolume soft terms in the presence of ISD fluxes is a special feature of D3-branes, and does not hold for other D-branes. For instance, it has been argued [17] that D7-branes in compactifications of the kind

\footnotetext{
${ }^{10}$ In this respect, in [40] stacks of anti-D3-branes were replaced by D7-brane worldvolume antiinstantons, whose tension couples as D-terms.

${ }^{11}$ In a sense, this is related to the instability issues: for instance, D-branes stuck at singularities avoid this problem because they may not contain the corresponding scalars.
} 
in [14] with ISD 3-form fluxes, have their moduli stabilized by the ISD flux. That is, the flux induces non-trivial mass terms for the D7-brane worldvolume scalars.

Clearly one can apply our general techniques to analyze the D7-brane worldvolume soft terms, again by simply coupling their DBI+CS action to the supergravity background. We leave this general problem for future research, but here provide the main features of soft terms for the interesting case of D7-branes in flat space, in the presence of ISD fluxes.

The effect of the background (3.10) on a D7-brane probe is quite simple. Indeed, since a D7-D3 system is BPS, and the ISD flux mimics the dilaton, metric and 5-form background of a D3-brane, it is clear that the dilaton, metric and 5-form in (3.11) lead to no soft terms on the D7-brane worldvolume. This follows from cancellations between diverse pieces in its action.

The only difference between an ISD and a D3-brane background is the presence of $G_{3}$ itself in the former. Hence, the soft terms we expect on D7-branes in ISD fluxes are those soft terms generated by $G_{3}$ directly, without use of the supergravity equations of motion. For instance, one immediate possible effect is the appearance of fermion masses, and scalar trilinear terms, in analogy with the above.

In addition and interestingly enough, there exists a new kind of soft term, namely scalar masses directly induced by the 3-form fluxes. The new contribution arises from the D7-brane DBI determinant, the analog of the last line in (2.8), involving the NSNS 2 -form field in worldvolume directions transverse to $4 \mathrm{~d}$ Minkowski space (denoted $a, b=$ $4,5,6,7)$

$$
\left[-\operatorname{det}\left(P\left[E_{\mu \nu}\right]\right)\right]^{1 / 2}=1-\frac{\sigma^{2}}{2} \partial_{\mu} \phi^{m} \partial^{\mu} \phi^{m}+\sigma^{2} K_{m n} \phi^{m} \phi^{n}-\frac{1}{2} B_{a b} B^{a b}
$$

Using that $B_{a b}=\frac{1}{3} H_{a b r} x^{r}+\ldots$, with $r=8,9$, and trading $x^{r}$ for $\phi^{r}$, we obtain additional soft terms involving masses for $\phi^{r}$

$$
\frac{1}{18} H_{a b r} H_{s}^{a b} \phi^{r} \phi^{s}
$$

Notice that there are no additional terms of this kind from the CS action, hence this contribution does not cancel out, and provides the non-zero scalar masses for D-brane worldvolume scalars, even for ISD fluxes.

The spacetime interpretation of this result is that if D7-branes move in transverse space, an induced D5-brane charge appears due to the non-trivial $B_{N S}$ field. This increases their tension, hence the D7-brane motion is energetically costly ${ }^{12}$. The

\footnotetext{
${ }^{12}$ We thank S. Trivedi and P. Tripathy for suggesting this mechanism.
} 
above scalar mass term accounts precisely for this additional tension, as is obvious from its DBI origin.

A more general argument, described in the literature, exploits the description of CY threefold compactifications with 3-form fluxes and 7-branes in terms of F-theory (of M-theory in a dual picture) on an elliptic CY fourfold with 4-form fluxes. In the latter, 7-branes are encoded in complex structure parameters, describing the geometry of the degenerations of the elliptic fibration, and which are fixed by the 4-form fluxes. Hence one concludes that 7-brane moduli are fixed by 3-form fluxes.

Notice that the existence of mass terms for D7-brane worldvolume fields for ISD fluxes does not spoil the no-scale structure of the resulting $4 \mathrm{~d}$ effective theory. Indeed, the masses of the D7-brane moduli are given by the flux density scale, hence is of the same order as masses of the complex structure moduli. This is also clear in the F-theory picture, where both kinds of moduli are on an equal footing.

\section{D3-branes at orbifolds}

Our results so far have been concerned with configurations of D3-branes in flat space, or at a smooth point in the transverse space. Consequently the D3-brane worldvolume field theory is a deformation of the $\mathcal{N}=4$ theory, and hence is non-chiral.

One of the natural ways to apply our results to the phenomenologically interesting setup of soft terms in chiral supersymmetric gauge sectors, would be to consider the D3-branes to sit at a singular point in transverse space. Moreover, one of the simplest choices of singularities, namely orbifold singularities, are just quotients of flat space by a discrete subgroup $\Gamma$ of the $S O(6)$ rotational isometry group in transverse space. Correspondingly the D3-brane worldvolume theory (denoted orbifold gauge field theory for short) is obtained from the underlying $\mathcal{N}=4 U(N)$ theory by projecting out fields non-invariant under such action [41]. In this projection, one should take into account (on top of the action on Chan-Paton (CP) labels) that the gauge bosons are singlets under the $S O(6)$, while scalars and fermions transform in the representations $\mathbf{6}, \mathbf{4}$.

The introduction of fluxes in the setup of D3-branes at singularities is straightforward, by regarding the configuration in the covering space. In the latter, we have D3-branes in a smooth supergravity background, on which only $\Gamma$-invariant supergravity fields are allowed. On the covering space, they induce a set of soft terms for the fields in the underlying parent $\mathcal{N}=4$ theory, as above. Due to the $S O(6)$ invariance of the above soft terms, the $\Gamma$-invariant supergravity background induces soft terms for 
T-invariant worldvolume fields, i.e. fields which descend to the orbifold field theory. Now going to the quotient, this implies that the supergravity background around the orbifold singularity induces the above soft terms in the orbifold gauge field theory. Notice that $S O(6)$ invariance is a crucial ingredient, guaranteeing that certain inconsistent soft terms (like mass terms for the chiral fermions present after the orbifold projection) are not present because the corresponding flux is projected out by the orbifold.

A particularly simple case is that of Abelian orbifold $\mathbf{C}^{\mathbf{3}} / \mathbf{Z}_{\mathbf{N}}$ singularities. The twist is given by a generator $\theta$ acting like

$$
\theta:\left(z_{1}, z_{2}, z_{3}\right) \rightarrow\left(e^{i 2 \pi a_{1} / N} z_{1}, e^{i 2 \pi a_{2} / N} z_{2}, e^{-i 2 \pi a_{3} / N} z_{3}\right)
$$

which leads to one unbroken SUSY on the D3-brane worldvolume (before adding fluxes) if $a_{1}+a_{2}+a_{3}=0, \bmod N$. If all $a_{i} \neq N / 2$, only fluxes of types $(0,3)$ and $(3,0)$ will be invariant under the twist. On the other hand, if one has e.g., $a_{3}=N / 2$ some primitive $(2,1)$ and $(1,2)$ fluxes will be consistent with the twist.

These features are more concretely discussed in explicit examples. Consider the $\mathbf{C}^{\mathbf{3}} / \mathbf{Z}_{\mathbf{3}}$ singularity, where the generator $\theta$ of $\mathbf{Z}_{\mathbf{3}}$ acts as

$$
\theta:\left(z_{1}, z_{2}, z_{3}\right) \rightarrow\left(e^{2 \pi i / 3} z_{1}, e^{2 \pi i / 3} z_{2}, e^{-4 \pi i / 3} z_{3}\right)
$$

Before the introduction of fluxes, the orbifold gauge field theory has the following $\mathcal{N}=1$ multiplet content

$$
\begin{array}{ccc}
V & \text { Vect. Mult. } & U(n) \times U(n) \times U(n) \\
\Phi_{a, a+1}^{1} & \text { Ch. Mult. } & (n, \bar{n}, 1)+(1, n, \bar{n})+(\bar{n}, 1, n) \\
\Phi_{a, a+1}^{2} & \text { Ch. Mult. } & (n, \bar{n}, 1)+(1, n, \bar{n})+(\bar{n}, 1, n) \\
\Phi_{a, a+1}^{3} & \text { Ch. Mult. } & (n, \bar{n}, 1)+(1, n, \bar{n})+(\bar{n}, 1, n)
\end{array}
$$

The allowed fields in the supergravity background are $K_{i \overline{\mathrm{j}}}, \tau_{i \overline{\mathrm{j}}}, \chi_{i \overline{\mathrm{j}}}$, and $G_{123}, G_{\overline{1} \overline{\overline{2}} \overline{3}}$, denoted $G_{0}, G_{0}{ }^{\prime}$ respectively in what follows.

So, due to the orbifold projection, the most general supergravity background is of the form considered in section 3.1.3. The soft terms are therefore

$$
\begin{aligned}
\mathcal{L}= & \operatorname{Tr}\left[-\left(2 K_{i \overline{\mathrm{j}}}-\chi_{i \overline{\mathrm{j}}}+g_{s}(\operatorname{Im} \tau)_{i \overline{\mathrm{j}}}\right) \Phi^{i} \Phi^{\overline{\mathrm{j}}}\right. \\
& \left.+\frac{g_{s} \sqrt{2 \pi}}{3} G_{0} \epsilon_{i j k} \Phi^{i} \Phi^{j} \Phi^{k}+\text { h.c. }+\frac{g_{s}^{1 / 2}}{2 \sqrt{2}} G_{0} \lambda \lambda+\text { h.c. }\right]
\end{aligned}
$$

where it is implicit that only gauge invariant combinations are present, namely $\Phi^{i} \Phi^{\bar{j}}$ stands for $\sum_{a} \Phi_{a, a+1}^{i}\left(\Phi_{a, a+1}^{j}\right)^{*}$ 
Notice that in particular no mass terms for the fermions in the chiral multiplets have been generated. Hence we see a nice interplay of the orbifold projection in the worldvolume field theory (where it leads to chirality via projection from the parent $\mathcal{N}=4$ theory) and in the supergravity background (where it forbids a background for non-invariant fields), correlated via the $S O(6)$ invariance of the underlying theory.

The above discussion can be carried out similarly for other orbifolds. It is interesting to notice that when the orbifold field theory contains non-chiral fermions, the fluxes that generate soft masses for them are invariant under the orbifold action. For instance, for orbifolds $\mathbf{C}^{\mathbf{2}} / \mathbf{Z}_{\mathbf{N}} \times \mathbf{C}$, with the $\mathbf{Z}_{\mathbf{N}}$ generated by $\theta:\left(z_{1}, z_{2}, z_{3}\right) \rightarrow$ $\left(e^{2 \pi i / N} z_{1}, e^{-2 \pi i / N} z_{2}, z_{3}\right)$, the worldvolume theory has $\mathcal{N}=2$ before the introduction of fluxes. Hence, the $\mathcal{N}=1$ chiral multiplet $\Phi_{3}$ contains adjoint fermions, $\Psi_{3}$, which are non-chiral. Correspondingly, one can consider $\mathbf{Z}_{\mathbf{N}}$-invariant IASD fluxes of the form

$$
G_{3}=G_{123} d z_{1} d z_{2} d z_{3}+G_{\overline{1} \overline{2} 3} d \bar{z}_{1} d \bar{z}_{2} d z_{3}
$$

which generically introduce soft terms breaking the $\mathcal{N}=2$ D3-brane worldvolume supersymmetry; in particular, the second term introduces a soft mass for $\Psi_{3}$.

An interesting example, leading to a chiral spectrum which contains a non-chiral subsector (which can thus acquire mass terms) is provided by the $\mathbf{C}^{\mathbf{3}} / \mathbf{Z}_{\mathbf{4}}$ orbifold, generated by $\theta:\left(z_{1}, z_{2}, z_{3}\right) \rightarrow\left(e^{2 \pi i / 4} z_{1}, e^{2 \pi i / 4} z_{2},-z_{3}\right)$.

The spectrum of $\mathcal{N}=1$ multiplets is given by ${ }^{13}$

$$
\begin{array}{ccc}
V & \text { Vect. Mult. } & U(n)_{1} \times U(n)_{2} \times U(n)_{3} \times U(n)_{4} \\
\Phi_{a, a+1}^{1,2} & \text { Ch. Mult. } & 2\left[\left(\bar{n}_{1}, n_{2}\right)+\left(\bar{n}_{2}, n_{3}\right)+\left(\bar{n}_{3}, n_{4}\right)+\left(\bar{n}_{4}, n_{1}\right)\right. \\
\Phi_{a, a+2}^{3} & \text { Ch. Mult. } & \left(n_{1}, \bar{n}_{3}\right)+\left(\bar{n}_{1}, n_{3}\right)+\left(n_{2}, \bar{n}_{4}\right)+\left(\bar{n}_{2}, n_{4}\right)
\end{array}
$$

The allowed non-trivial supergravity background parameters are $K_{i \bar{j}}$, for $i, j=1,2$, $K_{33}, K_{\overline{3} \overline{3}}, K_{3 \overline{3}}$ (and similar ones for $\tau_{m n}$ and $\xi_{m n}$ ), and $G_{123}, G_{\overline{1} \overline{2} \overline{3}}, G_{12 \overline{3}}, G_{\overline{1} \overline{2} 3}$. Thus, the most general allowed soft term lagrangian is

$$
\begin{aligned}
\mathcal{L} & =\operatorname{Tr}\left(-\sum_{i j=1,2}\left(2 K_{i \bar{\jmath}}-\chi_{i \bar{\jmath}}+g_{s}(\operatorname{Im} \tau)_{i \bar{\jmath}}\right) \Phi^{i} \Phi^{\bar{j}}-\frac{g_{s}^{1 / 2}}{2 \sqrt{2}}\left(G_{123} \lambda \lambda+\frac{1}{2} S_{33} \Psi^{3} \Psi^{3}\right)+\right.\text { h.c. } \\
& -\frac{1}{2}\left(2 K_{33}+\chi_{33}+g_{s}(\operatorname{Im} \tau)_{33}\right) \Phi^{3} \Phi^{3}+\text { h.c. }-\left(2 K_{3 \overline{3}}-\chi_{3 \overline{3}}+g_{s}(\operatorname{Im} \tau)_{3 \overline{3}}\right) \Phi^{3} \Phi^{\overline{3}} \\
& \left.+\frac{g_{s} \sqrt{2 \pi}}{3}\left[G_{123} \epsilon_{i j k} \Phi^{i} \Phi^{j} \Phi^{k}+\frac{3}{2} \epsilon_{\bar{i} \bar{j} \overline{3}} S_{33} \Phi^{\bar{i}} \Phi^{\bar{j}} \Phi^{3}\right]+\text { h.c. }\right)
\end{aligned}
$$

\footnotetext{
${ }^{13}$ We consider D3-branes in the regular representation of $\mathbf{Z}_{\mathbf{4}}$, although slightly more general choices can be made, consistently with twisted RR tadpole cancellations.
} 
where again it is implicit that only gauge invariant terms appear, namely $\Phi^{3} \Phi^{3}$ denotes couplings $\Phi_{1,3}^{3} \Phi_{3,1}^{3}+\Phi_{2,4}^{3} \Phi_{4,2}^{3}$ (and similarly for $\Psi^{3} \Psi^{3}$ ).

As we already mentioned, one interesting feature of this example is that the orbifold symmetry is compatible with flux components that lead to $\mu$-terms and B-terms for some worldvolume scalars. Indeed one has a $\mu$-term for the $\Psi^{3}$ fermion $\mu_{33}=$ $g_{s}^{1 / 2} S_{33} /(2 \sqrt{2})$. The B-terms arise arise from the coefficients $K_{33}, \tau_{33}, \chi_{33}$ for the $10 \mathrm{~d}$ metric, dilaton and 5 -form background ${ }^{14}$. It is interesting to point out that B-terms do not contribute to the trace of the mass matrix, and therefore are completely unrelated to the flux background, and depend crucially on the boundary conditions for fields in our local configuration (i.e. depend on the structure of distant sources or the global properties of the compactification). It is tempting to speculate that this may lead to interesting mechanisms to generate phenomenologically viable values for the B-term in MSSM-like models.

Finally we would like to point out that modding out the above $\mathbf{Z}_{\mathbf{3}}$ theory by the group of permutations of the three complex planes leads to a configuration of D3-branes at the $\Delta_{27}$ singularity $[7,8]$. One immediately recovers the background, field theory and soft terms reproducing ${ }^{15}$ those in [21]. Our approach in this paper is more general, and allows to study D3-branes at smooth points, and at other orbifolds. It would be interesting to device a way of computing soft terms for D3-branes at other interesting, but non-orbifold, singularities, like conifolds and generalizations thereof.

\section{$7 \quad$ SUSY-breaking soft terms and the effective $\mathcal{N}=1$ supergravity effective action}

The approach to compute soft terms we followed up to now was very general in the sense that we were able to compute them in terms of the local Type IIB supergravity backgrounds. In general we would be interested in full-fledged compact vacuum solutions, but in order to compute soft terms in the worldvolume of D3-branes that local information was enough.

In a full compactification we would like to interpret those terms as arising from vevs for the auxiliary components of some $4 \mathrm{~d}$ chiral superfields. In a general CY

\footnotetext{
${ }^{14}$ Note that, as emphasized, this kind of soft terms will arise in the worldvolume of anti-D3-branes in the presence of ISD $(0,3)$ and $(2,1)_{P}$ fluxes also. In particular, SUSY-preserving $(2,1)_{P}$ fluxes give rise to no soft terms on the worldvolume of $D 3$-branes but give rise to a ' $\mu$-term' on that of antibranes.

${ }^{15}$ Our results slightly correct those in [21] in a few numerical coefficients.
} 
compactification the natural candidates for such chiral fields will be the dilaton $S$ and Kähler and complex structure moduli. A simple class of models are the type IIB Calabi-Yau orientifold models in $[13,14]$ (which include the explicit $\mathbf{T}^{6} / \Omega R$ models in $[15,16,18]$, and others [17]). These authors showed that the presence of $\mathrm{RR}$ and NSNS fluxes is able to fix the values of the dilaton and generically all complex structure moduli, while Kähler moduli are in general not fixed. Our analysis in this section will apply to any such CY orientifold in the large radius limit, in which the behaviour of the Kähler potential is quite general.

\subsection{SUSY-breaking soft terms and $\mathcal{N}=1$ auxiliary fields}

In a general CY (not $\mathbf{T}^{\mathbf{6}}$ or $\mathrm{K} 3 \times \mathbf{T}^{\mathbf{2}}$ ) there are no non-trivial 5-forms. This implies that it is not possible to turn on non-primitive fluxes, since $G_{3} \wedge J \neq 0$ would define a nontrivial 5-form. Our analysis will center on general CY compactifications, hence the ISD condition will allow for $(0,3)$ and primitive $(2,1)$ pieces, and the IASD condition will allow for $(3,0)$ and primitive $(1,2)$ pieces. The analysis for $\mathbf{T}^{\mathbf{6}}$ or $\mathrm{K} 3 \times \mathbf{T}^{\mathbf{2}}$ is complicated due to the presence of additional supersymmetry and hence additional auxiliary fields. See $[19,18]$ for some discussion of $\mathbf{T}^{\mathbf{6}}$ compactification.

The massless spectrum in any CY-orientifold always includes the complex dilaton chiral field

$$
S=-i \tau ; \tau=C+i / g_{s},
$$

whose imaginary part is related to the dilaton and $C$ is the type IIB axion. In addition there will be a number of complex structure scalars $M_{a}$ and Kähler moduli $T_{\alpha}(18+18$ in the $T^{6} / \Omega R$ example of $\left.[15,16]\right)$, characterizing the compact volume. In order to simplify matters we will focus first on the dynamics of the dilaton chiral field $S$ and the overall Kähler modulus $T$, whose real part gives the overall radius of the compactification. More precisely (see e.g. refs.[14, 42, 18]), we will have $T=\exp 4 \sigma+i b$, with $\exp \sigma$ being the breathing mode of the compact manifold volume. In the large volume limit the Kähler potential has the well known form

$$
\frac{K}{M_{p}^{2}}=-\log \left(S+S^{*}\right)-3 \log \left(T+T^{*}\right)
$$

where $M_{p}^{2}$ is the $4 \mathrm{~d}$ Planck mass squared. We would now like to use the effective lagrangian in order to reproduce some of the results obtained for soft terms in the previous sections. We consider, as in $[13,14,15,16]$, compactifications with a nontrivial background for the NSNS and RR field strength 3-forms $H_{(3)}, F_{(3)}$. In this 
situation an $S$-dependent effective superpotential is given by [43]

$$
W=\kappa_{10}^{-2} \int G_{(3)} \wedge \Omega, G_{(3)}=F_{(3)}-i S H_{(3)},
$$

where $\kappa_{10}^{2}=\frac{1}{2}(2 \pi)^{7} \alpha^{4}$ is the $D=10$ gravitational constant and $\Omega$ the Calabi-Yau holomorphic 3-form.

The supergravity auxiliary field for a chiral field with Kähler metric $K_{i \overline{\mathrm{j}}}$ is given by

$$
\bar{F}^{\bar{i}}=\exp \left(K / 2 M_{p}^{2}\right) K^{\overline{i j}} D_{j} W / M_{p}^{2}
$$

with indices meaning derivation, and $D_{j} W=\partial_{j} W+K_{j} W$. Applying this to the $S$ and $T$ fields, one finds using the above expression for the superpotential,

$$
\begin{aligned}
& F^{S}=\frac{1}{M_{p}^{2}}\left(S+S^{*}\right)^{1 / 2}\left(T+T^{*}\right)^{-3 / 2}\left(\kappa_{10}^{-2}\right) \int G_{(3)}{ }^{*} \wedge \Omega \\
& F^{T}=-\frac{1}{M_{p}^{2}}\left(S+S^{*}\right)^{-1 / 2}\left(T+T^{*}\right)^{-1 / 2}\left(\kappa_{10}^{-2}\right) \int G_{(3)} \wedge \Omega
\end{aligned}
$$

Note that these equations show that a flux background of $(3,0)$ type would then correspond to a non-vanishing auxiliary field for the complex dilaton $S$, whereas a $(0,3)$ background would correspond to the overall Kähler field $T$. One also finds for the gravitino mass

$$
m_{3 / 2}^{2}=\frac{e^{K / M_{p}^{2}}}{M_{p}^{4}}|W|^{2}=\frac{1}{M_{p}^{4}}\left(S+S^{*}\right)^{-1}\left(T+T^{*}\right)^{-3}\left(\kappa_{10}^{-4}\right)\left|\int G_{(3)} \wedge \Omega\right|^{2}
$$

One can then compute the vacuum energy in terms of the auxiliary fields to be given by

$$
\begin{aligned}
V_{0} & =M_{p}^{2}\left(F^{S^{*}} K_{S S^{*}} F^{S}+F^{T^{*}} K_{T T^{*}} F^{T}\right)-3 m_{3 / 2}^{2} M_{p}^{2}=M_{p}^{2} F^{S^{*}} K_{S S^{*}} F^{S}= \\
& =\frac{\kappa_{10}^{-4}\left|\int G_{(3)}^{*} \wedge \Omega\right|^{2}}{M_{p}^{2}\left(S+S^{*}\right)\left(T+T^{*}\right)^{3}}
\end{aligned}
$$

As expected, the contribution of the $T$ auxiliary field to the vacuum energy precisely cancels the $-3 m_{3 / 2}^{2}$ supergravity term. This is just the well known cancellation taking place in no-scale models ${ }^{16}$. Thus only the complex dilaton auxiliary field contributes to the vacuum energy. Note that if one minimizes the energy, as would be required by the equations of motion, the dynamics of the system yield $\int G_{(3)}^{*} \wedge \Omega=0$, so that the $(3,0)$ piece vanishes, in agreement with the results in [14]. (In order to eliminate other IASD components, minimization with respect to complex structure moduli should be imposed as well, see below).

\footnotetext{
${ }^{16}$ This structure presumably does not survive $\alpha^{\prime}$ and $g_{s}$ corrections (see [44] for a discussion of some $\alpha^{\prime}$ correction terms).
} 
In addition to the dilaton and moduli fields, these compactifications include gauge sectors on D3-branes, which generically sit at smooth points (or less generically at a singular point) in the internal space. We would like to analyze, using the effective lagrangian approach, the structure of the SUSY-breaking soft terms induced by the fluxes. For such purpose we can use standard $\mathcal{N}=1$ supergravity formulae, already employed in the past to study soft terms in heterotic vacua (see ref.[45] for a review).

Masses for the gauginos of the $a^{\text {th }}$ gauge factor may be obtained from the general formula

$$
M_{a}=\left(2 \operatorname{Re} f_{a}\right)^{-1} F^{i} \partial_{i} f_{a}
$$

where $f_{a}$ is the gauge kinetic function. In the present D3-brane case this gauge kinetic function is simply given by $f_{a}=S$, so that one easily finds for gaugino masses

$$
M_{a}=\frac{1}{M_{p}^{2}}\left(S+S^{*}\right)^{-1 / 2}\left(T+T^{*}\right)^{-3 / 2}\left(\kappa_{10}^{-2}\right) \int G_{3}^{*} \wedge \Omega
$$

One can also use general expressions to compute soft terms trilinear in the adjoint scalars $\Phi_{i}$. For this we need to know the Kähler potential $K$ of the scalar fields. In this simplified model with only one diagonal overall Kähler field $T$, the Kähler potential $K$ for any such field is given by $K=1 /\left(T+T^{*}\right)$. The trilinear scalar soft terms are (see e.g. $[45])$

$$
\begin{aligned}
A_{i j k} & =h_{i j k}\left(F^{S} K_{S}+F^{T} K_{T}-\partial_{T} \log \left(K_{i} K_{j} K_{k}\right)\right)=h_{i j k} F^{S} K_{S}= \\
& =-h_{i j k} \frac{1}{M_{p}^{2}}\left(S+S^{*}\right)^{-1 / 2}\left(T+T^{*}\right)^{-3 / 2}\left(\kappa_{10}^{-2}\right) \int G_{3}^{*} \wedge \Omega=-h_{i j k} M^{a} .
\end{aligned}
$$

Note that the computation of gaugino masses and trilinear terms reproduce our previous results obtained from the DBI and CS pieces of the D3-brane action. Indeed, in the limit or large volume $V$, the quantity $\kappa_{10}^{-2} \int G_{3}^{*} \wedge \Omega$ becomes $\kappa_{10}^{-2} G_{0} \times V=M_{p}^{2} G_{0}$. On the other hand the prefactor scales like $g_{s}^{1 / 2} /\left(\sqrt{2} M_{p}^{2}\right)$, so that indeed one obtains $|M| \rightarrow \frac{g_{s}^{1 / 2}}{\sqrt{2}} G_{0}$, in agreement with the results obtained in section 2 .

Hence we recover the fact that the $(3,0)$ component of $G_{3}$ corresponds to a vev for the auxiliary field of the dilaton, and reproduces soft terms of the dilaton domination kind; exactly as found in the explicit discussion from the D3-brane worldvolume viewpoint.

On the other hand, for the scalar masses one obtains

$$
\begin{aligned}
m_{j}^{2} & =m_{3 / 2}^{2}+\frac{V_{0}}{M_{p}^{2}}-\sum_{i} F^{\bar{i}} F^{i} \partial_{\bar{i}} \partial_{i} \log \left(K_{j}\right)= \\
& =m_{3 / 2}^{2}+\frac{V_{0}}{M_{p}^{2}}-m_{3 / 2}^{2}=\frac{\kappa_{10}^{-4}\left|\int G_{(3)}^{*} \wedge \Omega\right|^{2}}{M_{p}^{4}\left(S+S^{*}\right)\left(T+T^{*}\right)^{3}}=\left|M_{a}\right|^{2}
\end{aligned}
$$


Notice that this does not agree with the DBI+CS result (3.12). Indeed this is expected: Our local configuration in section 2 assumed Poincare invariance in the four longitudinal directions. Hence the soft terms (3.12) correspond to those on configuration with $(3,0)$ flux and Poincare invariance. This cannot be reproduced in a flux compactification of the kind described by the above effective lagrangian, since non-ISD fluxes (like a $(3,0)$ component) always generate a non-zero vacuum energy (cosmological constant) that is not consistent with $4 \mathrm{~d}$ Poincare invariance. In this sense, the result (7.11) should correspond to the soft terms obtained from the DBI+CS action for D3-branes in a modified background, allowing for deviations from Poincare invariance.

Notice that in heterotic analysis $[2,3,4]$, dilaton dominated SUSY-breaking by $F_{S}$ was argued to correspond to the soft terms (3.12). This however does not contradict our above comment, since in those heterotic references the vacuum energy was not computable in the underlying theory (since no microscopic SUSY-breaking source was proposed), and it was imposed by hand that it vanishes. If this were the situation, the ansatz in section 2 would be a good local description, and soft terms would agree. However, in flux compactification the vacuum energy is computable and non-vanishing for non-ISD fluxes, leading to the above mentioned result.

Note that, if we insist in imposing the equations of motion in the flux compactification, one has $G_{(3)}^{*} \wedge \Omega=0$, which forces the ISD condition, and we recover the well known fact that in no-scale models all soft terms identically vanish, to leading order 17. One can also check that the field $T$ remains massless, whereas the complex dilaton field gets a mass equal to the gravitino mass (7.6).

It is also important to emphasize that the analysis in section 2 is more general than pure flux compactification of the kind in [14]. For instance, one expects that a fully realistic model (involving additional ingredients) should reproduce a tiny cosmological constant (hence essentially enjoying 4d Poincare invariance) while the supersymmetry breaking sources (e.g. 3-form fluxes) are still large, and induce large soft masses. In this kind of model, the local description should be precisely of the form in section 2 . This perfectly illustrates the usefulness of the analysis, and shows that the underlying difficulty to reconcile the local ansatz with a full-fledge compactification is the familiar difficulty to construct an explicit compactification with large SUSY-breaking and tiny vacuum energy.

One could similarly analyze the SUSY-breaking due to vevs for auxiliary fields of other moduli, namely the complex structure moduli $M_{a}$. These are on an equal footing

\footnotetext{
${ }^{17}$ See footnote 16.
} 
with respect to the dilaton in many aspects, but their couplings to the gauge sector fields is more model dependent, hence our discussion is more sketchy.

The complex structure moduli $M_{a}$ have a Kähler potential

$$
K(M, \bar{M})=-\log \left(-i \int_{X} \Omega \wedge \bar{\Omega}\right)
$$

with the dependence on the complex structure moduli is implicit in $\Omega$. Namely, for the complex structure modulus $M_{a}$, we have $\partial_{a} \Omega=k_{a} \Omega+\chi_{a}$, where $k_{a}$ is moduli dependent, and the set of $\chi_{a}$ is a basis of $(2,1)$ forms. We then have

$$
\partial_{a} K=-k_{a} \quad ; \quad \partial_{a} \partial_{\bar{b}} K=-\frac{\int_{X} \chi_{a} \wedge \bar{\chi}_{b}}{\int_{X} \Omega \wedge \bar{\Omega}}
$$

Using the superpotential $W=\int_{X} G_{3} \wedge \Omega$, the auxiliary field in the multiplet of $M_{a}$ is essentially given by

$$
D_{a} W=\partial_{a} W+W \partial_{a} K=\int_{X} G_{3} \wedge \chi_{a}
$$

Choosing the set of moduli to have diagonal metric, the auxiliary fields are very simple. The auxiliary field for $M_{a}$ is the piece of $G_{3}$ that wedges non-trivially with the corresponding $(2,1)$ form $\chi_{a}$. Clearly, only $(1,2)$ pieces of $G_{3}$ contribute to auxiliary fields of the complex structure moduli. In a general CY case the $(1,2)$ piece is primitive, and hence we see that only the IASD piece of $G_{3}$ contribute to the auxiliary field vevs. In $\mathbf{T}^{\mathbf{6}}$ or $\mathrm{K} 3 \times \mathbf{T}^{\mathbf{2}}$, the non-primitive $(1,2)$ piece of $G_{3}$ seems to lead to vevs for auxiliary fields as well. Since these pieces are ISD, their contribution to the supergravity scalar potential must however be cancelled by additional negative pieces e.g. from the supergravity multiplet auxiliary fields, in analogy with the cancellation for $(0,3)$ components (indeed both cancellations are related by the enhanced supersymmetries of the underlying compactification), thereby preserving the no-scale structure (see $[19,18]$ for a discussion in $\mathbf{T}^{\mathbf{6}}$ ).

The computation of the explicit soft terms from this approach is again affected by the issue of the non-zero vacuum energy in compactifications with this kind of flux background. Hence it differs from the results from the local analysis in section 3.1 .2 by reasons already explained.

In conclusion, the low energy $4 \mathrm{~d}$ supergravity approach allows us to identify the breaking of supersymmetry as a spontaneous breaking due to vevs for auxiliary fields of certain $4 \mathrm{~d}$ supermultiplets. 


\subsection{Soft terms and S-duality}

The underlying Type IIB supergravity theory enjoys the property of $S L(2, \mathbf{Z})$ S-duality. Under transformations given in terms of a matrix $m=\left(\begin{array}{ll}a & b \\ c & d\end{array}\right)$ with integer entries and unit determinant, the 3 -form $G_{(3)}$ transforms as

$$
G_{(3)} \rightarrow G_{(3)}^{\prime}=\frac{G_{(3)}}{i c S+d}
$$

and the dilaton transforms as

$$
i S \rightarrow \frac{i a S+b}{i c S+d} .
$$

Interestingly the flux-induced superpotential transforms like a modular form of weight -1 , i.e.

$$
W(S) \rightarrow \frac{W(S)}{i c S+d}
$$

From this it is clear that the complete Kähler potential $K+\log |W(S)|^{2}$ is invariant under S-duality, since the transformation of the $-\log \left(S+S^{*}\right)$ piece is cancelled by the transformation of the $S$-dependent superpotential. This works as in the first proposal of the existence of S-duality in string theory in [23].

Note in particular that due to this fact, all SUSY-breaking quantities, like the gravitino mass, are invariant under $D=4$ S-duality transformations, even though no unbroken SUSY in general remains. Similarly the SUSY-breaking soft terms felt by anti-D3-branes in the presence of $(0,3)$ backgrounds will be invariant as well.

\subsection{Finiteness properties of dilaton-domination and hologra- phy}

It was found by Jones, Mezincescu and Yao [27] ${ }^{18}$, long time before the idea of dilaton domination was considered, that the choice of soft terms $A_{i j k}=-h_{i j k} M$ and $3 m^{2}=M^{2}$ can be added to the $\mathcal{N}=4$ finite theory without spoiling finiteness. In $[24,25,26]$ it was noticed that dilaton domination soft terms precisely corresponded to those boundary conditions. Since then it has remained unclear why dilaton domination soft terms have this property.

Our explicit realization of soft terms in string theory interestingly provides a new insight into this question. Consider a configuration of purely $(3,0) G_{3}$ flux background in flat space. The full supergravity solution (exact when regarded as an expansion in the flux density) is, as discussed in section (2), of the black 3-brane form (3.9).

\footnotetext{
${ }^{18}$ For more recent discussion on these finiteness properties see e.g. [25, 28, 29].
} 
Notice in particular that the IIB complex coupling does not vary over spacetime. Now introduce a stack of D3-branes probing the background. From the point of view of the $4 \mathrm{~d}$ worldvolume gauge field theory, the flux background induces supersymmetry breaking soft terms of the dilaton-dominated kind (i.e. supersymmetry is broken by a vev for the auxiliary field which is partner of the dilaton). As we said, these soft terms preserve the finiteness of the theory. Moreover, the above results suggest that the gauge coupling is a marginal operator, on which the type IIB $S L(2, \mathbf{Z})$ acts as the S-duality group, leaving soft terms invariant.

The string theory construction allows to provide a simple interpretation for this result. It is known that the dependence of the IIB complex coupling on the coordinates transverse to the D3-brane reproduces the running of the field theory gauge coupling constant with the renormalization group scale. Hence in our situation the constant value of the dilaton suggests the softly broken theory still is finite, at least has vanishing beta function in perturbation theory. Moreover, the string theory construction captures the marginality of the coupling parameter and the action of S-duality.

A more precise form of the statement could be achieved by using the AdS/CFT description of the system, namely taking the near horizon limit of the configuration of D3-branes in the flux background. However the full supergravity solution for the combined system is not known so it is difficult to extract precise conclusions at this point.

We would like to finally point out an interesting observation. It was shown in $[28,29]$ that the finiteness condition for the softly broken $\mathcal{N}=4$ theory are slightly more general, and read

$$
m_{1}^{2}+m_{2}^{2}+m_{3}^{2}=M^{2} \quad ; \quad A_{i j k}=-h_{i j k} M
$$

where the $m_{i}$ are soft masses for the complex scalars in the three $\mathcal{N}=1$ chiral multiplets in $\mathcal{N}=4$. Hence, the finiteness property only constrains the trace of the mass matrix and allows the individual eigenvalues to change.

From our discussion after (3.8) and in appendix B.2, it is clear that this kind of deviation from mass universality (without change in the trace of the mass matrix) is present if the configuration contains additional sources, located away from the D3branes, besides the fluxes themselves. For instance, soft terms of the form (7.18) arise in configurations with pure $(3,0) G_{3}$ flux, and a set of anti-D3-branes located away form the D3-branes. The warp and 5-form background created by the $\overline{\mathrm{D} 3}$-branes superimposes over the flux background, and leads to changes in the scalar masses, 
keeping the trace of the mass matrix fixed (to the value $M^{2}$ ). It would be interesting to describe the finiteness of the resulting theory using holographic ideas in this more general backgrounds.

\section{A realistic orientifold with flux-induced soft terms}

As we have emphasized, the method presented to compute soft terms may be applied in a variety of situations. In particular, one can consider explicit compactifications where the above structures of soft terms are realized. A large class of type IIB Calabi-Yau or F-theory compactifications was introduced in [14], with O3-planes, D3-branes and ISD fluxes. From the viewpoint of soft terms this is a bit unfortunate, since as mentioned above ISD fluxes lead to cancellations between the DBI and CS parts of the D3-brane action, and produce no soft terms whatsoever. This agrees with the no-scale structure of the models.

One can still use these compactifications to study soft terms induced on the worldvolume of anti D3-branes. As discussed above, ISD fluxes do generate non-trivial soft terms on the latter. In fact, if the flux is purely $(0,3)$ the soft terms on the $\overline{\mathrm{D} 3}$-brane are of the dilaton-domination type. These $\overline{\mathrm{D} 3}$-branes can be regarded as probes of the configuration ${ }^{19}$. Interestingly this kind of situation also arises in the recent constructions of de Sitter vacua in string theory [20] (see also [40] for further developments), where the compactification includes fluxes that overshoot the RR tadpole from the background (e.g. O3-planes), and is compensated by the introduction of $\overline{\mathrm{D} 3}$-branes (which are also responsible for lifting the vacuum energy and leading to the de Sitter vacuum).

In the following we describe an explicit example, in which we have a local configuration with a stack of anti-D3-branes on top of a $\mathbf{Z}_{\mathbf{3}}$ singularity. The resulting theory has a realistic Left-Right symmetric $S U(3) \times S U(2)_{L} \times S U(2)_{R} \times U(1)_{B-L}$ gauge group on the worldvolume of the antibranes ${ }^{20}$. In order to cancel RR twisted tadpoles at this orbifold point we introduce an additional stack of anti-D7-branes passing through the singularity. The resulting theory has three quark-lepton generations and three sets

\footnotetext{
${ }^{19}$ One may worry about the 4-form tadpole they generate; one can assume we simultaneously introduce an additional D3-brane somewhere else in the internal space. Alternatively, one may introduce just $\overline{\mathrm{D} 3}$-branes, and fluxes, see the main text.

${ }^{20}$ It is equally easy to obtain similar models with the SM gauge group, see ref.[7]. We have chosen here a left-right symmetric model because the Chan-Paton matrices are simpler in this case.
} 
of Higgs doublets ${ }^{21}$. Moreover soft terms of the dilaton-dominated type appear.

The model is a simple modification of the left-right symmetric model in section 4.2.1 of the second ref.[7]. One just replaces branes by antibranes in that model, except for the D3-branes at the origin which remain unaltered. Untwisted tadpole cancellation will require the presence of 3 -form fluxes. We briefly review its construction in what follows.

Consider type IIB compactified on $\mathbf{T}^{\mathbf{6}} / \mathbf{Z}_{\mathbf{3}}$, where the orbifold is generated by the twist

$$
\theta \quad: \quad\left(z_{1}, z_{2}, z_{3}\right) \longrightarrow\left(e^{2 \pi i / 3} z_{1}, e^{2 \pi i / 3} z_{2}, e^{-4 \pi i / 3} z_{3}\right)
$$

Let us mod out by the orientifold action $\Omega R(-1)^{F_{L}}$, where $R: z_{i} \rightarrow-z_{i}$, and introduce a $G_{3}$ flux of the form

$$
G_{3}=A d \bar{z}_{1} d \bar{z}_{2} d \bar{z}_{3}
$$

which fixes the dilaton vev to $\tau=i S=e^{2 \pi i / 3}$. The coefficient $A$ is an even number to ensure proper quantization over toroidal 3-cycles ${ }^{22}$. The flux is purely $(0,3)$ and breaks supersymmetry, but is ISD and obeys the equations of motion. Its contribution to the 4-form tadpole is $N_{\text {flux }}=3|A|^{2}$.

There are 27 fixed points, which we label by $(m, n, p)$, where $m, n, p=0, \pm 1$, as shorthand for the three possible positions of the fixed points in each complex plane. At the fixed point $(0,1,0)$ (and its orientifold mirror $(0,-1,0))$ we locate $7 \overline{\mathrm{D} 3}$ 's with Chan-Paton (CP) matrices:

$$
\gamma_{\theta, \overline{3}}=\operatorname{diag}\left(\mathrm{I}_{3}, \alpha \mathrm{I}_{2}, \alpha^{2} \mathrm{I}_{2}\right)
$$

(see fig.1). In order to cancel twisted tadpoles at the fixed points $(0, n, p)$ we add 6 anti-D7-branes passing through them, with CP matrix

$$
\gamma_{\theta, \overline{7}}=\operatorname{diag}\left(\mathrm{I}_{2}, \alpha \mathrm{I}_{2}, \alpha^{2} \mathrm{I}_{2}\right)
$$

We furthermore add a Wilson line $\gamma_{W}$ on them on the second complex plane, given by

$$
\gamma_{W, \overline{7}}=\operatorname{diag}\left(\alpha, \alpha^{2}, \mathrm{I}_{2}, \mathrm{I}_{2}\right)
$$

\footnotetext{
${ }^{21}$ The model is a variant of that presented in section 4.2 .1 of the second ref.[7]. In addition ISD fluxes are introduced as in appendix $\mathrm{A}$ in [46].

${ }^{22}$ The space $\mathbf{T}^{\mathbf{6}} / \mathbf{Z}_{\mathbf{3}}$ does not have collapsed 3-cycles, hence there are no further subtleties in the flux quantization conditions, as compared with the toroidal case.
} 


\section{7 fixed points}
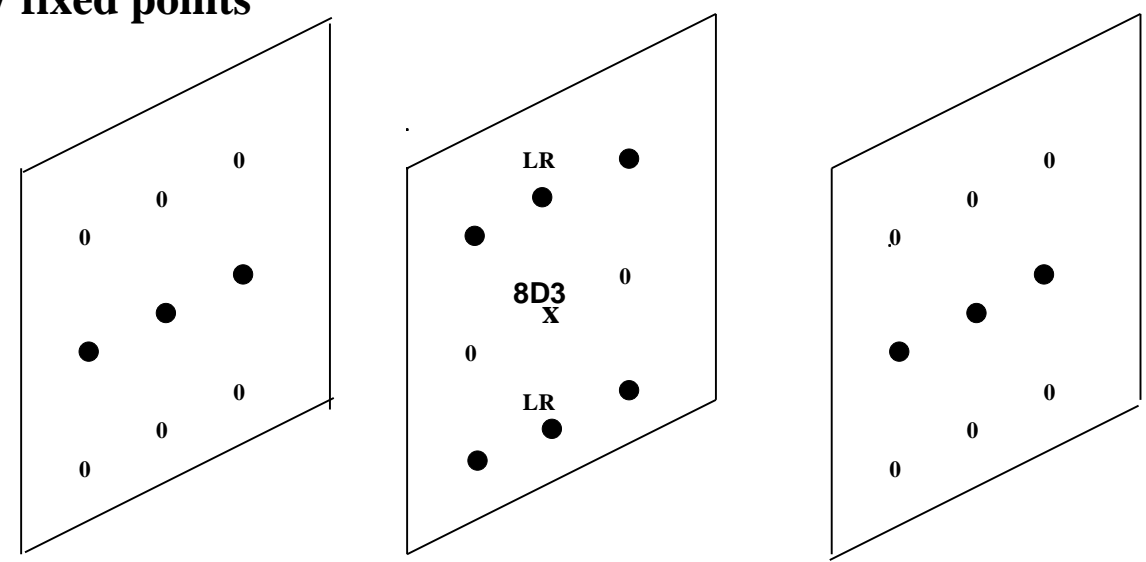

3 D7

$6 \mathrm{D7}^{*}$

3 D7

$X_{1}=-1$

$\mathbf{X}_{1}=\mathbf{0}$

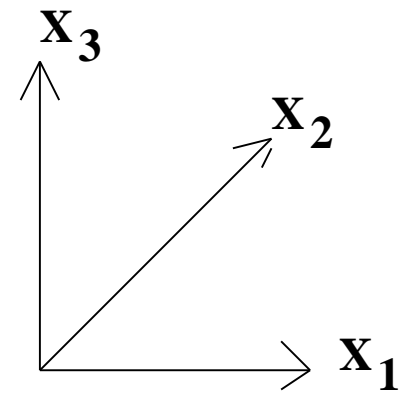

Figure 1: A compact Type IIB $T^{6} / \mathbf{Z}_{\mathbf{3}}$ orientifold model with a three generation $S U(3) \times$ $S U(2)_{L} \times S U(2)_{R} \times U(1)_{B-L}$ gauge theory. Here $X_{i}$ represent the three complex compact dimensions. The gauge theory lives on the worldvolume of $7 \overline{\mathrm{D} 3}$ 's located at the fixed point marked LR (and its orientifold mirror). The rest of the black dots represent one $\overline{\mathrm{D} 3}$. Upon switching on a self-dual $(0,3)$ flux RR-tadpoles cancel and SUSY-breaking soft terms appear on the worldvolume of the LR branes. 
In this way the gauge group coming from the $\overline{\mathrm{D} 7}$ 's is broken to $U(2) \times U(1)$ and the different fixed points have now different $\overline{\mathrm{D} 7}$-brane $\mathrm{CP}$ matrices. We complete a consistent configuration, cancelling all RR twisted tadpoles, as follows (see fig.1). We locate one $\overline{\mathrm{D} 3}$-brane at each of the four fixed points $(0, \pm 1, \pm 1)$ with $\mathrm{CP}$ matrix $\gamma_{\theta, \overline{3}}=1$, and 8 D3-branes at the origin, with $\mathrm{CP}$ matrix $\gamma_{\theta, 3}=\operatorname{diag}\left(\alpha \mathrm{I}_{4}, \alpha^{2} \mathrm{I}_{4}\right)$. Finally, in order to cancel global RR tadpoles, we add $3 \overline{\mathrm{D} 3}$ 's as well as 3 parallel $D 7$-branes (and their orientifold mirrors) passing respectively through the fixed points of type $(1, n, p)$ and $(-1, n, p)$ (see ref.[7] for details). One can easily check that all twisted tadpoles cancel in this configuration.

The total untwisted RR 4-form charge in the configuration is

$$
Q_{R R}=-32(O 3)-24\left(D 3^{*}\right)+8(D 3)=-48
$$

This charge is neatly cancelled if we add $\operatorname{ISD}(0,3)$ flux with $A=4$, which contributes to the RR-charge $3 \times(4)^{2}=48$ units.

This brane configuration is (meta)stable. The D7 and $\overline{\mathrm{D} 7}$ 's are stabilized on the planes passing through the orbifold points. They are forced to remain there in order to maintain twisted RR tadpole cancellation. Equivalently, the orbifold projection removes the scalar associated to flat directions describing brane motion. This will avoid D7- $\overline{\mathrm{D} 7}$ annihilation. The addition of fluxes will not destabilize them, since their only effect would be to generate a potential for those scalars, if they were present.

The same happens for the $\overline{\mathrm{D} 3}$ 's whose scalars get masses of the dilaton dominated type. The D3-branes at the origin do not get masses from fluxes, since they feel the ISD flux which gives no soft terms (to leading order) for them. They are however stuck at the origin again by the twisted RR tadpole conditions, which would be violated if any of those branes traveled to the bulk (equivalently, their worldvolume field theory does not contain scalars parametrizing the possibility of moving away the D3-branes). In fact the four $\overline{D 3}$ 's at the four $(0, \pm 1, \pm 1)$ fixed points are also stuck by twisted tadpole conditions. All in all, the whole brane configuration is (meta)stable due to a combination of trapping at the fixed points and flux-induced scalar potentials.

The complete spectrum of the left-right symmetric model of interest is given in section 4.2.1 of ref.[7]. The gauge group is $U(3) \times U(2) \times U(2)$, with two anomalous $U(1)$ 's being actually massive, and the diagonal combination giving $B-L$. In the $\overline{\mathbf{3 3}}$ sector, we obtain matter fields

$$
\overline{33} \text { sector : } \quad 3[(3, \overline{2}, 1)+(\overline{3}, 1,2)+(1,2, \overline{2})]
$$

corresponding to three left(right)-handed quarks $Q_{L}^{a}\left(Q_{R}^{a}\right), a=1,2,3$, and three sets of 
standard Higgs multiplets $H^{a}$. From the $\overline{\mathbf{3 7}}$ and $\overline{\mathbf{7 3}}$ sectors, one gets:

$$
\begin{array}{ll}
\overline{\mathbf{3 7}} \text { sector : } & (3,1,1 ; 1)_{-1}+(3,1,1 ; 2)_{0}+(1,2,1 ; 1)_{1}+(1,2,1 ; 2)_{0} \\
\overline{\mathbf{7 3}} \text { sector : } & (\overline{3}, 1,1 ; 1)_{1}+(\overline{3}, 1,1 ; 2)_{0}+(1,1,2 ; 1)_{1}+(1,1,2 ; 2)_{0}
\end{array}
$$

These contains three left (right)-handed leptons $L^{a}\left(R^{a}\right)$. There are also some extra vector-like pairs of colour triplets will in general become massive once $\left(7_{i} 7_{i}\right)$ states get vevs (see ref.[7]). The orientifold projection map the sets of branes at $(0,1,0)$ and $(0,-1,0)$ fixed points to each other, so only one copy of the LR model is obtained.

The quarks in this model have a superpotential

$$
W_{Y}=g \sqrt{2} \epsilon_{a b c} Q_{L}^{a} Q_{R}^{b} H^{c}
$$

On the other hand there are no renormalizable lepton Yukawas which may only appear after a blowing up of the singularity [7]. We will thus concentrate here on the quarks. The flux background is of ISD $(0,3)$ type, hence it leads to dilaton dominated soft terms on the worldvolume of the anti-D3-branes. As discussed in section 3.1.2, these are

$$
\begin{aligned}
m_{Q_{L}^{a}}^{2}=m_{Q_{R}^{a}}^{2}=m_{H^{a}}^{2} & =m_{a}^{2} a=1,2,3 \\
m_{1}^{2}+m_{2}^{2}+m_{3}^{2} & =\frac{g_{s}}{6}\left|G_{\overline{1} \overline{2} \overline{3}}\right|^{2} \\
M_{3}=M_{L}=M_{R}=M_{B-L}=M & =\frac{g_{s}^{1 / 2}}{\sqrt{2}} G_{\overline{1} \overline{2} \overline{3}} \\
A^{a b c} & =-h^{a b c} M
\end{aligned}
$$

with the rest of the soft terms vanishing. This kind of SUSY-breaking soft terms applied to the MSSM have been abundantly studied in the literature, and provides a phenomenologically interesting and viable soft term pattern [48, 4, 50, 49, 51].

In the above analysis we have ignored the effect of the closed string Kähler moduli, which are not stabilized in the present context, and would acquire runaway potentials due to the uncancelled tensions. This feature would clearly not be present in more complete models which could include a mechanism to stabilize Kähler moduli, like gauge non-perturbative effects [20], and a small cosmological constant. In that case configurations of the kind presented above would be metastable. Still, the soft terms felt by the SM fields would be essentially independent of the avatars of the stabilization, and would be essentially of the dilaton domination form shown above.

Clearly the above kind of construction can be carried out in other manifolds, and configurations. It is interesting that the general pattern applies to models closely related to the class of models leading to de Sitter compactifications. 


\section{Some phenomenological issues}

\subsection{Mass scales and the hierarchy}

The flux quantization conditions tell us that on any 3-cycle $\Pi$

$$
\frac{1}{2 \pi \alpha^{\prime}} \int_{\Pi} F_{3} \in 2 \pi \mathbf{Z} \quad ; \quad \frac{1}{2 \pi \alpha^{\prime}} \int_{\Pi} H_{3} \in 2 \pi \mathbf{Z}
$$

As discussed in [14], compactifications with 3-form fluxes do not stabilize the overall volume modulus $T$. Therefore the very low energy effective field theory generically contains it as a modulus, and its parameters are functions of it. In particular, soft terms depend on the volume modulus because, due to the above quantization conditions, the flux densities have the dependence for large radius

$$
G_{3}=f \frac{\alpha^{\prime}}{R^{3}}
$$

with $f$ an $R$-independent constant measuring the amount of quantized flux. This is the well know fact that flux effects are suppressed like $1 / R^{3}$. From previous sections we see that the typical scale for soft terms will then be

$$
m_{\text {soft }}=\frac{g_{s}^{1 / 2}}{\sqrt{2}} G_{3}=\frac{f g_{s}^{1 / 2}}{\sqrt{2}} \frac{\alpha^{\prime}}{R^{3}}
$$

This could be interesting because for large radii we can get a hierarchy of scales

$$
g_{s}^{1 / 2} \frac{\alpha^{\prime}}{R^{3}}(\text { fluxes }) \ll \frac{1}{R}(\mathrm{KK}) \ll M_{s}
$$

(with $M_{s}$ the string scale) and identify the lightest one with the electroweak scale. For sufficiently large $\mathrm{R}$ the tower of KK states may be ignored and we can discuss physics of fluxes using the $4 \mathrm{~d}$ effective field theory. This has been implicit in our discussion in section 7. On the other hand, the dependence of the Planck scale with the overall volume is given by

$$
M_{p}=\left(\alpha^{\prime}\right)^{-2} R^{3}
$$

and hence we have

$$
m_{\text {soft }}=\frac{f M_{s}^{2}}{M_{p}}
$$

with $M_{s}=\alpha^{\prime-1 / 2}$ the string scale. This expression, in the effective $\mathcal{N}=1$ supergravity language is consistent with some auxiliary field (like e.g. that of the dilaton ) getting a vacuum expectation value of order $f M_{s}^{2}$. Eq. (9.6) is an interesting expression. Note that if one wants to identify $m_{\text {soft }}$ with the electroweak scale, the string scale should 
be of order the intermediate scale, $M_{s}=10^{10} \mathrm{GeV}$, a possibility considered by some authors in the past [52]. Note that in order to get the correct value for $M_{p}$ the compact radii should be of order $R=10^{3} \sqrt{\alpha^{\prime}}$.

Note that in this scenario the SUSY-breaking scale is small compared to the string scale because the flux is diluted by the fact the the compactification radius is 3 orders of magnitude larger than the string length scale. This requires a choice of large overall Kähler modulus vev. Notice however that there are interestingly models where the latter is stabilized via non-perturbative effects, and used in the construction of de Sitter vacua in string theory [20] (see also [40]). Stabilization at such moderately large overall volumes is achieved with small fine-tuning. It would be interesting to find explicit vacua in which these features arise.

Note also that the above computation involved only the dependence on the overall scale, and assumed more or less homogeneously diluted 3-form fluxes. In particular it would be possible to consider situations where the D3-branes are located in a region of the internal space where the fluxes are hierarchically diluted as compared with other regions. One prototypical example would be Calabi-Yau's containing throats in which most of the flux is concentrated [53, 14]. D3-branes away from the throat would feel the flux in a very suppressed manner, and the soft terms would be affected by additional suppression factors, as compared with the above estimate. This would correspond to a suppressed coefficient $f$ in eq.(9.6). In this case part (or all) of the weak scale versus Planck scale suppression could be due to this effect and the string scale could be closer (or identified) with the Planck scale. On the other hand the inverse situation is possible. If D-branes are located in a region of compact space with much more flux than the average, the string scale could be lower than the intermediate scale. In this setup, the largeness of the Planck mass would not be due to a large size for the extra dimensions, but rather to their strong warping [56, 14].

Let us finally comment that in the early days of heterotic, it was soon realized that a 3-form flux background for $H_{i j k}$ would break supersymmetry $[54,55]$. However it was noted in [57] that its flux was quantized ${ }^{23}$ so that the scale of SUSY-breaking would be necessarily of order of the Planck scale (recall that in the perturbative heterotic case $M_{p}$ is directly related to $M_{S}$ and that making compact radii very large leads to a difficult regime of strong coupling). Type II compactifications provide the new possibility discussed above, compact radii may be large in a consistent manner, and D3-branes lead to localized gauge sectors, sensitive to the flux density rather than to

\footnotetext{
${ }^{23}$ See [58] for a recent improvement of the situation.
} 
the integral of the flux. Thus the setup allows for hierarchically small breaking of SUSY, if desired.

\subsection{The structure of soft terms and its application to the SUSY SM}

It is conceivable that the soft terms of a completely realistic version of the MSSM embedded in a Type IIB vacuum could have their origin in the supergravity backgrounds that we are considering. The details of those terms would be model dependent, but one can advance some general properties of the soft terms that one would expect to appear if SUSY-breaking has the origin considered in this paper. Some general properties are the following:

i) Gaugino masses and trilinear A-terms

These only appear (in leading order) if a background for $G_{3}$ is present. This is important for model-building since present limits on SUSY particles already require the presence of substantial gaugino masses. The relationship $A=-h M$ found from flux induced SUSY-breaking is quite model independent. In as much as one can associate the heaviest SM generation Yukawa coupling as coming from a truncation of an underlying $\mathcal{N}=4$ theory (which happens in many realistic compactifications constructed up to now) one would expect the constraint $A_{t}=-h_{t} M$ to be quite generic. Here $A_{t}$ is the trilinear term associated to the top quark Yukawa coupling.

ii) Scalar $m^{2}$ terms

Whereas gaugino masses and trilinear terms depend only on the local background $G_{3}$ around the location of the branes, scalar masses also depend on other backgrounds (5-form and dilaton) as well as on the form of the warping. Hence they are sensitive to other distant sources (like other branes or antibranes, orientifold planes etc.). Thus scalar masses will depend on details of the full vacuum solution.

In general, soft scalar masses are different for different scalar fields. Thus in a realistic compactifications different e.g., squark flavors will have different masses. However, for configurations and backgrounds with certain geometrical symmetries (like the spherically symmetric cases mentioned e.g. in subsection 3.1.2, leading to dilaton dominated type of soft terms) scalars may all have identical masses. This issue is important phenomenologically, since there are strong constraints on mass differences, e.g. among the first and second generation squarks in the MSSM, coming from sufficient suppression 
of Flavor Changing Neutral Currents (FCNC).

Note that distant sources like e.g., antibranes give rise on the worldvolume of branes to D-term type of breaking, i.e. scalar masses but neither gaugino masses nor trilinear terms. This is in general insufficient for getting a phenomenologically acceptable SUSY-breaking.

iii) $\mu$-term

As we saw in the specific $\mathbf{Z}_{\mathbf{4}}$ example in section 6 , fluxes provide a new way to obtain a ' $\mu$-term' in string theory. The obtained term is proportional to the primitive component of $(1,2)$ antisymmetric fluxes $\left(S_{i j}\right.$ components). It is natural in flux induced SUSY-breaking that all fluxes (either e.g. $(3,0)$ leading to gaugino masses and $A$-parameters as well as $(1,2)$ type, leading to a $\mu$-term) to be of the same order $\propto g_{s}^{1 / 2} \alpha^{\prime} / R^{3}$. Thus the present scheme provides a simple solution to the so called ' $\mu$-problem', which is understanding why a SUSY-term like $\mu$ is of the same order of magnitude as SUSY-breaking soft terms. They simply have similar origin.

iv) B-term

The present scheme gives also a natural source for the presence of a B-term in the theory. It depends on the warping, 5-form and dilaton backgrounds and hence it is more model-dependent than the rest of the terms. Furthermore, its value is not fixed by the value of antisymmetric fluxes via the local supergravity equations of motion. Nevertheless one would expect its size to be of the order of the other soft terms, as can be estimated in explicit examples.

\section{v) Complex phases}

In general the soft $A, M_{a}, \mu$ and $B$ parameters are complex. The phases of the first three are related to the (generically complex) $G_{3}$ backgrounds. In some particular cases (like e.g. when $G_{3}=G_{3}^{*}$, corresponding to vanishing NSNS 3-form flux) they may become real. The $B$ parameter is generically complex and unrelated to the flux phases. All these phases are relevant for the so called SUSY CP-problem, which is that arbitrary complex soft terms give rise to $\mathrm{CP}$-violating transitions (electric dipole moment of the neutron), with rates three orders of magnitude larger than the experimental bounds. In the MSSM the relevant phases for these transitions are $\phi_{A}=\arg \left(M_{a} A^{*}\right)$ and $\phi_{B}=\arg \left(M B^{*}\right)$. Note that in fluxed induced SUSY-breaking one has $\phi_{A}=0$ and there is no $\mathrm{CP}$-violating contribution from that source. On the other hand the value 
of $\phi_{B}$ will depend on the phases of $B$ and $M_{a}$ which are in general uncorrelated. On the other hand, by appropriately choosing the form of the warping, 5-form and dilaton backgrounds $\left(K_{i j}, \chi_{i j}\right.$ and $\operatorname{Im} \tau_{i j}$ in eq.(2.22)) one can always get a vanishing $\phi_{B}$.

The above considerations seem to suggest the necessity of considering explicit antisymmetric $G_{3}$ background and negligible effects from distance sources on the SM particles, in order to get a phenomenologically viable model. In order to obtain sufficient degeneracy among squarks, some geometrical symmetry should be present.

\section{Final comments}

In the present paper we have computed the SUSY-breaking soft terms on the worldvolume of D3-branes induced by a general class of Type IIB supergravity backgrounds including RR and NS 3-form fluxes. The supergravity backgrounds are expanded around the location of the D3-branes, and the lowest terms in this expansion correspond precisely to the lowest dimensional SUSY-breaking soft terms. Different patterns of SUSY-breaking soft terms are obtained depending on the tensorial structure of the antisymmetric $G_{3}$ backgrounds. The results are summarized in eqs.(2.22). The computation applies to $D 3$-branes sitting on a smooth point in compact case but also to D3 branes sitting on e.g., orbifold singularities leading to chiral theories with phenomenological interest.

This approach is quite general in the sense that it allows us to compute SUSYbreaking terms induced by a general supergravity background. Thus for example, one can compute the breaking induced by distant $\overline{D 3}$-branes or orientifold planes. It also applies to explicit compactifications in which one can treat the physics around the D3-brane position locally. In compactifications the flux-induced SUSY-breaking terms may be interpreted as arising from vevs of the auxiliary fields of the complex dilaton $S$, the Kähler and complex structure moduli. This is somewhat analogous (though not identical) to the results for soft terms obtained in heterotic $[2,3,4,45]$ and Type I [5, 59] schemes. While the soft terms obtained from $(0,3)$ or $(3,0)$ do indeed correspond to the no-scale and dilaton domination soft terms in those references, general combinations of ISD and IASD fluxes lead to new possibilities not previously considered in heterotic schemes. One of the new interesting aspects is the generation of both $B$ and $\mu$-terms in a novel way.

One interesting question is the structure of mass scales introduced by flux SUSY- 
breaking. In particular, one would like to identify the soft terms with those required by the MSSM for its viability. We find that soft masses are of order $M_{s}^{2} / M_{p}$, with $M_{s}$ the string scale and that in order to identify that with the electroweak scale one needs to have $M_{s}=10^{10} \mathrm{GeV}$. This is compatible with the observed value of the Planck scale if the compactification scale is three orders of magnitude smaller than the string scale. Note that in this scheme two effects are the cause of the large hierarchy between electroweak and Planck scales. A large compact volume increases the value of $M_{p}^{2}$ (which goes like $\kappa_{10}^{-2} \mathrm{Vol}$ ) whereas at the same time dilutes the flux-induced soft terms (which go like $\alpha^{\prime} / \mathrm{Vol}^{1 / 2}$ ). The combination of both effects gives rise to a large hierarchy although, as we said, the compact radius is only 3 orders of magnitude larger than the string length. On the other hand the presence of a large warp factor could also lead to the generation of the hierarchy.

Another interesting property is the fact that all soft terms so obtained are invariant under $D=4, S L(2, \mathbf{Z})$ S-duality acting on the complex dilaton and the three-form flux. One also gets an understanding of the finiteness of the dilaton-dominated soft terms when added to the $\mathcal{N}=4$ lagrangian, which arises from the fact that the dilaton is constant in the IASD $(3,0)$ background and holography.

Examples of CY compactifications with ISD $(0,3)$ fluxes were considered in [14], containing also O3-planes and D3-branes. Those compactifications have a no-scale structure which cancels soft terms to leading order. It is easy to modify those schemes in order to have non-vanishing (and interesting) SUSY-breaking soft terms by adding antiD3-branes to the compactification. The degrees of freedom on the worldvolume of $\bar{D} 3$ branes feel effective $(3,0)$ fluxes, leading to non-trivial soft terms. We have constructed an explicit $Z_{3}$ orientifold example with a $S U(3) \times S U(2)_{L} \times S U(2)_{R} \times U(1)_{B-L}$ three generation model in which this effect takes place. We believe it should be possible to construct in this way models in which all moduli are fixed yet non-trivial soft terms are obtained for the SM fields.

\section{Acknowledgments}

We thank J. F. G. Cascales, D. Cremades, D.R.T. Jones and F. Quevedo for useful discussions. L.E.I. and P.G.C. thank CERN's Theory Division for hospitality. This work has been partially supported by the European Commission under the RTN contract HPRN-CT-2000-00148 and the CICYT (Spain). The work of P.G.C. is supported by the Ministerio de Educación, Cultura y Deporte (Spain) through a FPU grant. 


\section{A SUSY-breaking soft terms}

We include in this section for reference some general definitions for soft terms which we employ in the main text. We are interested in gauge theories which can be written in some approximation as $\mathcal{N}=1$ SUSY theories with chiral fields $\phi_{i}, i=1, . . M$ transforming under some representation of the gauge group. Let the renormalizable superpotential have the general form

$$
W(\phi)=\frac{1}{3 !} h^{i j k} \phi_{i} \phi_{j} \phi_{k}
$$

The $\mathcal{N}=4$ case is a particular example with 3 adjoint chiral fields. There is a finite list of soft SUSY-breaking terms which may be added to the lagrangian without introducing quadratic divergences. One has

$$
L_{\text {soft }}=-\left(m^{2}\right)_{i j} \phi_{i} \phi_{j}^{*}-\left(\frac{1}{3 !} A^{i j k} \phi_{i} \phi_{j} \phi_{k}+\frac{1}{2} B^{i j} \phi_{i} \phi_{j}-\frac{1}{2} M^{a} \lambda_{a} \lambda_{a}+\text { h.c. }\right) .
$$

Here $\lambda_{a}$ is a gaugino field and $a$ runs over the different gauge group factors. One can also consider the addition of extra terms as in

$$
L_{\text {soft }}^{(2)}=-\frac{1}{2} \mu^{i j} \psi_{i} \psi_{j}+\frac{1}{2} C^{i j k} \phi_{i} \phi_{j}^{*} \phi_{k}^{*}+M_{g}^{i a} \psi_{i} \lambda_{a}+\text { h.c. }
$$

where $\psi_{j}$ denotes the fermionic partners of the $\phi_{j}$. The last term is only present if the matter field transforms in the adjoint. The other two terms may in fact lead to quadratic divergences (and hence they would not be soft) but such quadratic divergences are absent if the chiral multiplets are not singlet under the gauge group, so in full generality one should consider them [60]. In fact, in the case

$$
\left(m^{2}\right)_{i j}=\left|\mu^{i j}\right|^{2} ; C^{i j k}=-\left(h^{j k l}\right)^{*} \mu_{i l}
$$

with the rest of the soft terms vanishing, the theory becomes supersymmetric and is described by the above trilinear superpotential plus a a SUSY mass term $\frac{1}{2} \mu_{i j} \phi_{i} \phi_{j}$.

There are some choices of $\mathcal{N}=0$ soft terms which have particularly interesting ultraviolet properties. Consider in particular the case of $\mathcal{N}=4$ supersymmetry. It has been shown that the choice of soft terms

$$
A^{i j k}=-M h^{i j k} ; \operatorname{Tr}\left(m^{2}\right)_{i j}=|M|^{2}
$$

with the other terms vanishing is ultraviolet finite to all orders in perturbation theory $[27,25,28,29]$. It is interesting that if one assumes that the auxiliary field of the 
complex dilaton $S$ is the only source of SUSY-breaking in a $D=4$ heterotic compactification one gets the result:

$$
A^{i j k}=-\sqrt{3} m_{3 / 2} h^{i j k} ; M_{a}=\sqrt{3} m_{3 / 2} \quad m_{i}^{2}=m_{3 / 2}^{2}
$$

which is a particular case of eq.(A.5). Thus dilaton dominated SUSY-breaking applied to a $\mathcal{N}=4$ theory maintains finiteness to all orders.

\section{B More general backgrounds}

\section{B.1 Linear terms}

As discussed in the main text, our power-expansion of the background fields starts at the quadratic order. This is because linear terms in the metric, dilaton, $\chi_{4}, \ldots$ generically lead to terms linear in the scalar fields in the D3-brane worldvolume action. In this appendix we briefly describe this in more detail.

For instance consider a background metric with a linear term, for instance

$$
Z_{1}^{1 / 2}\left(x^{m}\right)=1+Z_{m} x^{m}+\ldots
$$

From the DBI action, the leading terms in the worldvolume scalar action are

$$
\mathcal{L}=\frac{1}{2} \partial_{\mu} \phi^{m} \partial^{\mu} \phi^{m}+2 Z_{m} \phi^{m}
$$

so that the configuration sits in the slope of the scalar potential. It may be possible

to consider configurations where the linear terms in the background expansion cancel out at the level of the scalar potential, but this situation is highly non generic.

It is easy to consider examples of backgrounds with such linear terms. Consider the D3-brane is embedded in the supergravity background created by a stack of anti D3-branes at a distant point $x_{0}^{m}$. The background is

$$
\begin{aligned}
d s^{2} & =Z\left(x^{m}\right)^{-1 / 2} \eta_{\mu \nu} d x^{\mu} d x^{\nu}+Z\left(x^{m}\right)^{1 / 2} d x^{m} d x^{m} \\
\chi_{4} & =\frac{1}{Z} d x^{0} d x^{1} d x^{2} d x^{3} \\
F_{5} & =d \chi_{4}+* d \chi_{4} \\
Z & =1-\frac{1}{2 \pi^{2}} \frac{N}{\left|\vec{x}-\overrightarrow{x_{0}}\right|^{4}}
\end{aligned}
$$

We have

$$
Z=\text { const. }-\frac{2 x_{0}^{m} N}{\pi^{2} r_{0}^{6}} x^{m}+\frac{N}{\pi^{2} r_{0}^{4}}\left[\frac{\delta_{m n}}{r_{0}^{2}}+6 \frac{x_{0}^{m} x_{0}^{n}}{r_{0}^{4}}\right] x^{m} x^{n} \ldots
$$


with $r_{0}^{2}=\sum_{n}\left(x_{0}^{n}\right)^{2}$. This leads to linear terms for $\phi^{m}$, making the configuration unstable. Recalling the interpretation of $\phi$ as coordinate of the D3-brane in transverse space, the instability makes the D3-brane move towards the point $\vec{x}_{0}$, i.e. towards the antibrane. Namely, this is the worldvolume derivation of the gravitational attraction felt by the D3-brane. There is a similar linear terms arising from the D3-brane interaction with the RR 5-form background created by the antibranes

\section{B.2 Scalar masses}

In this section we elaborate on the fact that the warp factor is not fully determined in terms of the fluxes unless some choice of boundary conditions is made. For instance, it is clear that to any solution for the equation of motion (3.3), (3.4) one can superimpose a solution of the homogeneous equations. For instance, we can superimpose the

$$
\begin{aligned}
Z & =\text { const. }-\frac{1}{2 \pi^{2}} \frac{1}{\left|\vec{x}-\overrightarrow{x_{0}}\right|^{4}}-\frac{1}{2 \pi^{2}} \frac{1}{\left|\vec{x}+\overrightarrow{x_{0}}\right|^{4}}= \\
& =\text { const. }+\frac{2}{\pi^{2} r_{0}^{4}}\left[\frac{\delta_{m n}}{r_{0}^{2}}+6 \frac{x_{0}^{m} x_{0}^{n}}{r_{0}^{4}}\right] x^{m} x^{n}
\end{aligned}
$$

In the patch $|\vec{x}|<r_{0}$ the above is a vacuum solution. The additional piece in the warp factor can be regarded as the metric background created by a pair of antibranes located at $\pm \overrightarrow{x_{0}}$ (so that the linear terms cancel out due to the $\mathbf{Z}_{\mathbf{2}}$ symmetric distribution), but it can be regarded as a vacuum solution in the local patch around the D3-branes (at $\vec{x}=0$ ). In the latter view, the additional piece of the warp factor modifies the behaviour of the metric background at the boundary of the patch.

In any of these views, it is clear that the additional background modifies the masses of the worldvolume scalars, by an amount which is completely unrelated to the 3-form fluxes present in the configuration. Notice that this is consistent with the equation of motion, and so implies that the additional piece of the mass matrix is traceless.

Hence, as mentioned in the main text, the expression of the scalar masses in terms of the background metric, dilaton and 5-form depends just on the local background for these fields. Now specifying a relation between the scalar masses and the fluxes involves a choice of boundary condition for the metric, dilaton and 5-form. In this sense, locality is more manifest in the expression of soft scalar masses in terms of the metric, dilaton, and 5-forms, without use of the equations of motion. 


\section{Conventions}

Throughout the paper we use a perturbed $S O(9,1)$ Minkowskian metric with 'mostly plus' signature, and antisymmetric tensor defined by $\epsilon_{0123456789}=1$. Indices $M, N, \ldots$ are $10 \mathrm{~d}$, indices $\mu, \nu, \ldots$ are $4 \mathrm{~d}$, and indices $i, j, \ldots$ run over the six dimensions transverse to the D3-brane.

In general we take the same conventions as in [14,61]. Hence, the type IIB supergravity action is given by (already in the Einstein frame)

$$
\begin{aligned}
S_{I I B}= & \frac{1}{2 \kappa^{2}} \int d^{10} x(-G)^{1 / 2}\left[R-\frac{e^{2 \phi}}{2} \partial_{M} \tau \partial^{M} \bar{\tau}-\frac{1}{12} e^{\phi} G_{3} \cdot \bar{G}_{3}-\frac{1}{480} F_{5}^{2}\right]+ \\
& +\frac{1}{8 i \kappa^{2}} \int e^{\phi} C_{4} \wedge G_{3} \wedge \bar{G}_{3}
\end{aligned}
$$

where

$$
\begin{array}{rlrl}
G_{3} & =F_{3}-\tau H_{3} & F_{3} & =d C_{2} \\
F_{5}=d C_{4}-\frac{1}{2} C_{2} \wedge H_{3}+\frac{1}{2} B_{2} \wedge F_{3} & \tau=C+i e^{-\phi} .
\end{array}
$$

Note that $C_{4}$ defined by (C.3) in general is different from $\chi_{4}$, defined in (2.6). However, since in our particular background $B_{2}$ and $C_{2}$ vanish in the worldvolume, we have that $\left(C_{4}\right)_{0123}=\left(\chi_{4}\right)_{0123}$.

From here we can derive the equations of motion and Bianchi identities. In particular, we will use the following ones

$$
\begin{aligned}
d F_{5}= & \frac{i}{2} g_{s} G_{3} \wedge \bar{G}_{3} \\
\nabla^{M}\left(\frac{i}{2} e^{\phi} \partial_{M} \tau\right)- & \frac{1}{2} e^{2 \phi} \partial^{M} C \partial_{M} \tau=\frac{g_{s}}{24} G_{M N P} G^{M N P} \\
R_{M N}= & \frac{1}{4} e^{2 \phi} \partial_{M} \tau \partial_{N} \tau^{*}+\frac{1}{4} e^{2 \phi} \partial_{M} \tau^{*} \partial_{N} \tau+\frac{g_{s}^{2}}{96} F_{M Q R S T} F_{N}{ }^{Q R S T} \\
& +\frac{g_{s}}{8}\left(G_{M}{ }^{P Q} G_{N P Q}^{*}+G_{N}{ }^{P Q} G_{M P Q}^{*}-\frac{1}{6} g_{M N} G_{P Q R}^{*} G^{P Q R}\right) \\
d * \operatorname{Re} G_{3}= & F_{5} \wedge H_{3} .
\end{aligned}
$$

where our prescription for the hodge dual operation * is the common one in supergravity

$$
* A_{\mu_{1} \ldots \mu_{d-p}}=\frac{1}{p !} \epsilon_{\mu_{1} \ldots \mu_{d-p}} \nu_{1 \ldots \nu_{p}} A_{\nu_{1} \ldots \nu_{p}}
$$

Note that our conventions are slightly different from the ones of [30]. In particular

$$
\begin{array}{cll}
\text { Myers } & & \text { Ours } \\
C_{4} & \rightarrow & C_{4}+(1 / 2) B_{2} \wedge C_{2} \\
B_{2} & \rightarrow & -B_{2}
\end{array}
$$


Hence Myers' action takes now the form

$$
\begin{aligned}
S=-\mu_{3} \int d^{4} \xi & \operatorname{Tr}\left[e^{-\phi} \sqrt{-\operatorname{det}\left(P\left[E_{\mu \nu}+E_{\mu i}\left(Q^{-1}-\delta\right)^{i j} E_{j \nu}\right]+2 \pi \alpha^{\prime} F_{\mu \nu}\right) \operatorname{det}\left(Q_{i j}\right)}+\right] \\
& +\mu_{3} \int \operatorname{Tr}\left(P\left[e^{2 \pi i \alpha^{\prime} \mathbf{i}_{\phi} \mathbf{i}_{\phi}}\left(\sum_{n} C_{n}+\frac{1}{2} B_{2} \wedge C_{2}\right) e^{-B}\right] e^{2 \pi \alpha^{\prime} F}\right)
\end{aligned}
$$

with the usual definitions for the pull-back, $Q_{i}{ }^{j}$ and

$$
E_{M N}=G_{M N}-B_{M N}
$$

The new term $B_{2} \wedge C_{2}$ will make no difference in our computations since its background will be null in the worldvolume in order not to violate Lorentz invariance.

We hope there is no confusion between the momentary double use of $\phi$, as in the dilaton $e^{-\phi}$, and as the worldvolume scalars $\phi_{m}$ in $\mathbf{i}_{\phi}$. In the main text, $\phi$ is used only for the latter purpose.

Consistently with the above, it is adequate to define $C_{6}$ through ${ }^{24}$

$$
-* \operatorname{Re} G^{(3)}=d C_{6}-H_{3} \wedge\left(C_{4}+\frac{1}{2} B_{2} \wedge C_{2}\right)
$$

In fact, we see that when we apply exterior derivative to (C.12) we recover the corresponding equation of motion (C.7).

Whenever we complexify the transverse 6 dimensions we will do it by means of

$$
\begin{aligned}
& z^{l}=\frac{1}{\sqrt{2}}\left(x^{2 l+2}+i x^{2 l+3}\right) \\
& \bar{z}^{l}=\frac{1}{\sqrt{2}}\left(x^{2 l+2}-i x^{2 l+3}\right)
\end{aligned}
$$

It is not hard to see that our metric, antisymmetric and delta tensors on this new basis take the form

$$
\begin{aligned}
& g_{\mu \nu}=\operatorname{diag}(-1,1,1,1)=-\eta_{\mu \nu} \\
& \epsilon_{\tilde{0} \tilde{1} \tilde{2} \tilde{3} 1 \overline{1} 2 \overline{2} 3 \overline{3}}=(-i)^{3}=i \quad ; \quad \epsilon_{1 \overline{1} 2 \overline{2} 3 \overline{3}}=i \quad ; \quad \epsilon_{123}=\epsilon_{\overline{1} \overline{2} \overline{3}}=1 \\
& g_{i j}=g_{\overline{i \bar{\jmath}}}=0 \quad ; \quad g_{i \bar{\jmath}}=g_{\overline{i j}}=1 \\
& \delta_{i \bar{i}}=\delta_{\overline{i i}}=1 \quad ; \quad \delta_{i i}=\delta_{\overline{i i}}=0
\end{aligned}
$$

where tilded indices mean worldvolume indices.

Note that $g_{\mu \nu}$ in (C.15) does not correspond to the usual choice in field theory where $\eta_{\mu \nu}=\operatorname{diag}(1,-1,-1,-1)$. Therefore, in the low energy effective action we must change the sign of the contractions of the form $A_{\mu} A^{\mu}$.

\footnotetext{
${ }^{24}$ See [31] for further details.
} 
The complex conjugate tensor is defined in complex coordinates by

$$
A_{i}^{*}=\left(A_{\bar{i}}\right)^{*} \quad ; \quad A_{\bar{i}}^{*}=\left(A_{i}\right)^{*}
$$

Recall finally that this is not the usual complexification of the Dirac algebra, which is defined with a $1 / 2$ factor, and hence the lowering and raising operators are given by

$$
\sqrt{2} \Gamma_{1}^{ \pm}=\frac{1}{\sqrt{2}}\left(\Gamma^{4} \pm i \Gamma^{5}\right) \quad ; \quad \sqrt{2} \Gamma_{2}^{ \pm}=\frac{1}{\sqrt{2}}\left(\Gamma^{6} \pm i \Gamma^{7}\right) \quad ; \quad \sqrt{2} \Gamma_{3}^{ \pm}=\frac{1}{\sqrt{2}}\left(\Gamma^{8} \pm i \Gamma^{9}\right)
$$

\section{References}

[1] For a review see e.g. F. Quevedo, 'Lectures on superstring phenomenology', hepth/9603074.

[2] L.E. Ibáñez and D. Lüst, 'Duality anomaly cancellation, minimal string unification and the effective low-energy Lagrangian of 4-D strings', Nucl. Phys. B382 (1992) 305, hep-th/9202046.

[3] V. S. Kaplunovsky, J. Louis, 'Model independent analysis of soft terms in effective supergravity and in string theory, Phys. Lett. B306 (1993) 269, hep-th/9303040.

[4] A. Brignole, L. E. Ibáñez, C. Muñoz, 'Towards a theory of soft terms for the supersymmetric Standard Model', Nucl. Phys. B422 (1994) 125, Erratum-ibid. B436 (1995) 747, hep-ph/9308271.

[5] L. E. Ibáñez, C. Muñoz and S. Rigolin, 'Aspects of Type I string phenomenology', Nucl. Phys. B553 (1999) 43, hep-ph/9812397.

[6] G. Aldazabal, L. E. Ibáñez, F. Quevedo, 'Standard - like models with broken supersymmetry from type I string vacua', JHEP 0001 (2000) 031, hep-th/9909172;

'A D-brane alternative to the MSSM', JHEP 0002 (2000) 015.

[7] G. Aldazabal, L. E. Ibáñez, F. Quevedo, A.M. Uranga, 'D-branes at singularities: A Bottom up approach to the string embedding of the standard model', JHEP 0008 (2000) 002, hep-th/0005067.

[8] D. Berenstein, V. Jejjala and R.G. Leigh, 'The standard model on a D-brane', Phys.Rev.Lett.88 (2002) 071602, hep-ph/0105042. 
[9] D. Bailin, G. V. Kraniotis and A. Love, "Searching for string theories of the standard model," hep-th/0108127;

L. L. Everett, G. L. Kane, S. F. King, S. Rigolin and L. T. Wang, "Supersymmetric Pati-Salam models from intersecting D-branes," hep-ph/0202100;

L. F. Alday and G. Aldazabal, "In quest of just the Standard Model on D-branes at a singularity," hep-th/0203129.

[10] R. Blumenhagen, L. Görlich, B. Körs and D. Lüst, "Noncommutative compactifications of type I strings on tori with magnetic background flux,” JHEP 0010, 006 (2000), hep-th/0007024; G. Aldazábal, S. Franco, L. E. Ibáñez, R. Rabadán and A. M. Uranga, " $D=4$ chiral string compactifications from intersecting branes," J. Math. Phys. 42, 3103 (2001), hep-th/0011073; "Intersecting Brane Worlds," JHEP 0102, 047 (2001), hep-ph/0011132; R. Blumenhagen, B. Körs and D. Lüst, “Type I strings with F- and B-flux," JHEP 0102, 030 (2001), hep-th/0012156;

L. E. Ibáñez, F. Marchesano and R. Rabadán, "Getting just the Standard Model at Intersecting Branes,” JHEP 0111, 002 (2001), hep-th/0105155.

[11] For some reviews on intersecting D-brane models see e.g. F. Marchesano, 'Intersecting D-brane models', hep-th/0307252; A. M. Uranga, 'Chiral four-dimensional string compactifications with intersecting D-branes,' Class. Quant. Grav. 20 (2003) S373, hep-th/0301032; R. Blumenhagen, B. Kors, D. Lüst, T. Ott,' Intersecting brane worlds on tori and orbifolds', hep-th/0112015; R. Blumenhagen, V. Braun, B. Körs, D. Lüst, 'The standard model on the quintic', hep-th/0210083; D. Cremades, L. E. Ibanez and F. Marchesano, 'More about the standard model at intersecting branes', arXiv:hep-ph/0212048; L. E. Ibanez, 'Standard model engineering with intersecting branes,' arXiv:hep-ph/0109082; M. Cvetic, 'Supersymmetric particle physics from intersecting D-branes', Proc. of SUSY02, vol. 1* 502511 (Hamburg, 2002); C. Kokorelis, '4-D GUT (and SM) model building from intersecting D-branes', hep-th/0310194; G. Honecker, 'Supersymmetric intersecting D6-branes and chiral models on the $T^{6} /\left(Z_{4} \times Z_{2}\right)$ orbifold', hep-th/0309158.

[12] J. Polchinski, A. Strominger, 'New vacua for type II string theory', Phys. Lett. B388 (1996) 736, hep-th/9510227;

K. Becker, M. Becker, 'M theory on eight manifolds', Nucl. Phys. B477 (1996) 155, hep-th/9605053;

J. Michelson, 'Compactifications of type IIB strings to four-dimensions with nontrivial classical potential', Nucl. Phys. B495 (1997) 127, hep-th/9610151; 
B. R. Greene, K. Schalm, G. Shiu, 'Warped compactifications in M and F theory', Nucl. Phys. B584 (2000) 480, hep-th/0004103;

G. Curio, A. Klemm, D. Lust, S. Theisen, 'On the vacuum structure of type II string compactifications on Calabi-Yau spaces with H fluxes', Nucl. Phys. B609 (2001) 3, hep-th/0012213;

M. Haack, J. Louis, 'M theory compactified on Calabi-Yau fourfolds with background flux', Phys. Lett. B507 (2001) 296, hep-th/0103068;

J. Louis, A. Micu, 'Type 2 theories compactified on Calabi-Yau threefolds in the presence of background fluxes', hep-th/0202168;

[13] K. Dasgupta, G. Rajesh, S. Sethi, 'M theory, orientifolds and G-flux', JHEP 9908 (1999) 023, hep-th/9908088.

[14] S. B. Giddings, S. Kachru, J. Polchinski, 'Hierarchies from fluxes in string compactifications', hep-th/0105097.

[15] A. R. Frey, J. Polchinski, 'N=3 warped compactifications', hep-th/0201029.

[16] S. Kachru, M. Schulz, S. Trivedi, 'Moduli stabilization from fluxes in a simple iib orientifold', hep-th/0201028.

[17] P. K. Tripathy, S. P. Trivedi, 'Compactification with flux on K3 and tori', hepth/0301139.

[18] M. Berg, M. Haack, B. Kors, 'An Orientifold with fluxes and branes via T duality', Nucl. Phys. B669 (2003) 3, hep-th/0305183.

[19] R. D'Auria, Sergio Ferrara, S. Vaula, 'N=4 gauged supergravity and a IIB orientifold with fluxes', New J.Phys. 4 (2002) 71, hep-th/0206241;

S. Ferrara, M. Porrati, 'N=1 no-scale supergravity from IIB orientifolds', Phys. Lett. B545 (2002) 411, hep-th/0207135;

R. D'Auria, S. Ferrara, M. Lledó and S. Vaulá, 'No-scale $N=4$ supergravity coupled to Yang-Mills: the scalar potential and super-Higgs effect', Phys.Lett.B557 (2003) 278, hep-th/0211027;

R. D'Auria, S. Ferrara, F. Gargiulo, M. Trigiante and S. Vaulá, ' $N=4$ supergravity Lagrangian for Type IIB on $T^{6} / Z_{2}$ orientifold in presence of fluxes and D3-branes', JHEP 0306:045,2003, hep-th/0303049; 
L. Andrianopoli, S. Ferrara and M. Trigiante, 'Fluxes, supersymmetry breaking and gauged supergravity', hep-th/0307139.

[20] S. Kachru, R. Kallosh, A. Linde, S. P. Trivedi, 'De sitter vacua in string theory', hep-th/0301240.

[21] M. Graña, 'MSSM parameters from supergravity backgrounds', hep-th/0209200.

[22] E. Cremmer, S. Ferrara, C. Kounnas and D.V. Nanopoulos, 'Naturally vanishing cosmological constant in $N=1$ supergravity', Phys.Lett.B133 (1983) 61.

[23] A. Font, L. E. Ibáñez, D. Lüst, F. Quevedo, 'Strong - weak coupling duality and nonperturbative effects in string theory', Phys. Lett. B249 (1990) 35.

[24] L.E. Ibáñez and C. Muñoz, unpublished (1994); C. Muñoz at the Lake Tahoe Conference 'Beyond the Standard Model IV', December 1994..

[25] I. Jack, D. R. T. Jones, 'Renormalization group invariance and universal soft supersymmetry breaking', Phys. Lett. B349 (1995) 294-299, hep-ph/9501395.

[26] L. E. Ibáñez, 'Strings, unification and dilaton / moduli induced SUSY breaking', proceedings of Strings 95: Future Perspectives in String Theory, hep-th/9505098.

[27] D. R. T. Jones, L. Mezincescu, Y. P. Yao, 'Soft breaking of two loop finite $N=1$ supersymmetric gauge theories', Phys. Lett. B148 (1984) 317-322

[28] T. Kobayashi, J. Kubo, G. Zoupanos, 'Further all-loop results in softly-broken supersymmetric gauge theories', Phys. Lett. B427 (1998) 291, hep-ph/9802267.

[29] I. Jack, D. R. T. Jones, A. Pickering, 'Renormalization invariance and the soft Beta functions', Phys. Lett. B426 (1998) 73, hep-ph/9712542.

[30] R. C. Myers, 'Dielectric branes', JHEP 9912 (1999) 022, hep-th/9910053.

[31] J. Polchinski, M. J. Strassler, 'The String dual of a confining four-dimensional gauge theory', hep-th/0003136.

[32] M. Cederwall, A. von Gussich, B. E. Nilsson, P. Sundell and A. Westerberg, 'The Dirichlet super-p-branes in ten-dimensional type IIA and IIB supergravity', Nucl. Phys. B 490 (1997) 179, hep-th/9611159;

M. Cederwall, A. von Gussich, B. E. Nilsson and A. Westerberg, 'The Dirichlet super-three-brane in ten-dimensional type IIB supergravity', Nucl. Phys. B 490 (1997) 163, hep-th/9610148. 
[33] M. Graña, 'D3-brane action in a supergravity background: The fermionic story', Phys. Rev. D 66 (2002) 045014, hep-th/0202118.

[34] D. Marolf, L. Martucci and P. J. Silva, 'Actions and fermionic symmetries for D-branes in bosonic backgrounds', JHEP 0307 (2003) 019, hep-th/0306066.

[35] M. Graña, J. Polchinski, 'Supersymmetric three form flux perturbations on $\mathrm{AdS}_{5}$ ', Phys. Rev. D63 (2001) 026001, hep-th/0009211.

[36] S. Kachru, J. Pearson and H. Verlinde, 'Brane/flux annihilation and the string dual of a non-supersymmetric field theory', JHEP 0206, 021 (2002), hep-th/0112197.

[37] D. Martelli, J. Sparks, 'G structures, fluxes and calibrations in M theory', Phys. Rev. D68 (2003) 085014, hep-th/0306225; A. R. Frey, M. Graña, 'Type IIB solutions with interpolating supersymmetries', Phys. Rev. D68 (2003) 106002, hepth/0307142; J. F.G. Cascales, A. M. Uranga, 'M5-brane geometries, T duality and fluxes', hep-th/0307156; K. Becker, M. Becker, R. Sriharsha, 'PP waves, M theory and fluxes', hep-th/0308014.

[38] I. Antoniadis, E. Dudas, A. Sagnotti, 'Brane supersymmetry breaking', Phys. Lett. B464 (1999) 38, hep-th/9908023;

G. Aldazabal, A. M. Uranga, 'Tachyon free nonsupersymmetric type IIB orientifolds via Brane - anti-brane systems', JHEP 9910 (1999) 024, hep-th/9908072.

[39] G. R. Dvali, Q. Shafi, S. Solganik, 'D-brane inflation', hep-th/0105203;

C. P. Burgess, M. Majumdar, D. Nolte, F. Quevedo, G. Rajesh, R.-J. Zhang, 'The Inflationary brane anti-brane universe', JHEP 0107 (2001) 047, hep-th/0105204.

S. Kachru, R. Kallosh, A. Linde, J. Maldacena, L. McAllister and S. Trivedi, 'Towards inflation in string theory', hep-th/0308055.

[40] C. Escoda, M. Gómez-Reino, F. Quevedo, 'Saltatory de Sitter string vacua', hepth/0307160; C. P. Burgess, R. Kallosh, F. Quevedo, 'De Sitter string vacua from supersymmetric D terms', hep-th/0309187.

[41] M. R. Douglas, G. W. Moore, 'D-branes, quivers, and ALE instantons', hep$\operatorname{th} / 9603167$.

[42] O. de Wolfe and S. Giddings, 'Scales and hierarchies in warped compactifications and brane worlds', Phys.Rev.D67 (2003) 066008, hep-th/0208123;

A. Frey and A. Mazumdar, '3-form induced potentials, dilaton stabilization and 
running moduli', Phys.Rev.D67:046006 (2003), hep-th/021025;

S. Giddings, 'The fate of four dimensions', Phys.Rev.D68:026006,2003, hepth/0303031;

A. Frey, 'Warped strings: self-dual flux and contemporary compactifications', hepth/0308156.

[43] S. Gukov, C. Vafa, E. Witten, 'CFT's from Calabi-Yau four folds', Nucl. Phys. B584 (2000) 69, Erratum-ibid. B608 (2001) 477, hep-th/9906070;

T. R. Taylor, C. Vafa, 'R R flux on Calabi-Yau and partial supersymmetry breaking', Phys. Lett. B474 (2000) 130, hep-th/9912152;

S. Gukov, 'Solitons, superpotentials and calibrations', Nucl. Phys. B574 (2000) 169, hep-th/9911011;

K. Behrndt, S. Gukov, 'Domain walls and superpotentials from M theory on Calabi-Yau three folds', Nucl. Phys. B580 (2000) 225, hep-th/0001082;

[44] K. Becker, M. Becker, M. Haack, J. Louis, 'Supersymmetry breaking and alphaprime corrections to flux induced potentials', JHEP 0206 (2002) 060, hepth/0204254.

[45] A. Brignole, L. E. Ibáñez, C. Muñoz, 'Soft supersymmetry breaking terms from supergravity and superstring models', in 'Perspectives on supersymmetry', G. Kane ed., World Scientific, hep-ph/9707209.

[46] J. F. G. Cascales, A. M. Uranga, 'Chiral 4d N=1 string vacua with D branes and NSNS and RR fluxes', hep-th/0303024.

[47] R. Blumenhagen, D. Lust, T. R. Taylor, 'Moduli Stabilization in Chiral Type IIB Orientifold Models with Fluxes', hep-th/0303016.

[48] R. Barbieri, J. Louis and M. Moretti, 'Phenomenological implications of supersymmetry breaking by the dilaton', Phys.Lett.B312(451)1993, hep-ph/9305262.

[49] J.A. Casas, A. Lleyda and C. Muñoz, 'Problems for supersymmetry breaking by the dilaton in strings from charge and color breaking', Phys.Lett.B380(1996)59, hep-ph/9601357.

[50] A. Brignole, L. E. Ibáñez, C. Muñoz, 'Orbifold induced $\mu$-term and electroweak symmetry breaking', Phys.Lett.B387(1996)769, hep-ph/9607405. 
[51] S. Abel, B. Allanach, F. Quevedo, L. E. Ibáñez and M. Klein, 'Soft SUSY-breaking, dilaton domination and intermediate scale string models', JHEP 0012:026,2000, hep-ph/0005262.

[52] K. Benakli, 'Phenomenology of low quantum gravity scale models', Phys. Rev. D60 (1999) 104002, hep-ph/9809582;

C .P. Burgess, L. E. Ib añez, F. Quevedo, 'Strings at the intermediate scale, or is the Fermi scale dual to the Planck scale?', Phys. Lett. B447 (1999) 257, hep$\mathrm{ph} / 9810535$.

[53] I. R. Klebanov, M. J. Strassler, 'Supergravity and a confining gauge theory: Duality cascades and chi SB resolution of naked singularities', JHEP 0008 (2000) 052, hep-th/0007191.

[54] J.P. Derendinger, L. E. Ibáñez and H.P. Nilles, 'On the low-energy $D=$ 4, $N=1$ supergravity theory extracted from the $D=10, N=1$ superstring', Phys.Lett.B155(1985)65.

[55] M. Dine, R.Rohm, N. Seiberg and E. Witten, 'Gluino condensation in superstring models', Phys.Lett.B156(1985)55.

[56] L.Randall, R. Sundrum, 'A Large mass hierarchy from a small extra dimension', Phys. Rev. Lett. 83 (1999) 3370, hep-ph/9905221.

[57] R. Rohm, E. Witten, 'The antisymmetric tensor fields in superstring theory', Ann. Phys. 170 (1986) 454.

[58] G.L. Cardoso, G. Curio, G. Dall'Agata and D. Lüst, 'Heterotic string theory on non-Kähler manifolds with H-flux and gaugino condensate', hep-th/0310021;

E. Buchbinder and B. Ovrut, 'Vacuum stability in heterotic M-theory', hepth/0310112;

S. Gukov, S. Kachru, X. Liu, L. McAllister, 'Heterotic moduli stabilization with fractional Chern-Simons invariants', hep-th/0310159.

[59] B. Körs and P. Nath, 'Effective action and soft supersymmetry breaking for intersecting D-brane models', hep-/th/0309167.

[60] I. Jack and D.R.T. Jones, 'Non-standard soft supersymmetry breaking', Phys.Lett.B457(1999)101, hep-ph/9903365. 
[61] J. Polchinski, 'String Theory. Vol. 2: Superstring Theory And Beyond', (Cambridge University Press, Cambridge, England, 1998) 